\title{
A dual-tube model for gas dynamics in fractured nanoporous shale formations
}

\author{
I. Lunati ${ }^{1} \dagger$ and S. H. Lee ${ }^{2}$ \\ ${ }^{1}$ Institute of Earth Sciences, University of Lausanne, Géopolis 3771, \\ UNIL-Mouline, 1015 Lausanne, Switzerland \\ ${ }^{2}$ Chevron Energy Technology Co., 1500 Louisiana Street, Houston, TX 77002, USA \\ (Received 13 February 2014; revised 11 July 2014; accepted 3 September 2014; \\ first published online 1 October 2014)
}

Gas flow through fractured nanoporous shale formations is complicated by a hierarchy of structural features (ranging from nanopores to microseismic and hydraulic fractures) and by several transport mechanisms that differ from the standard viscous flow used in reservoir modelling. In small pores, self-diffusion becomes more important than advection; also, slippage effects and Knudsen diffusion might become relevant at low densities. We derive a nonlinear effective diffusion coefficient that describes the main transport mechanisms in shale-gas production. In dimensionless form, this coefficient depends only on a geometric factor (or dimensionless permeability) and on the kinetic model that describes the gas. To simplify the description of the complex structure of fractured shales, we observe that the production rate is controlled by the flow from the shale matrix (which has the smallest diffusivity) into the fracture network, which is assumed to produce instantaneously. Therefore, we propose to model the flow in the shale matrix and estimate the production rate with a simple bundle-of-dual-tubes model (BoDTM), in which each tube is characterized by two diameters (one for transport and the other for storage). The solution of a single tube is approximately self-similar at early time, but not at late time, when the gas flux decays exponentially owing to the finite length of the tube. To construct a BoDTM, a reliable estimate of the joint statistics of the matrix-porosity parameters is required. This can be either inferred from core measurements or postulated on the basis of some a priori assumptions when information from laboratory and field measurements is scarce. By comparison with field production data from the Barnett shale-gas field, we demonstrate that BoDTM can be calibrated to estimate structural parameters of the shale formation and to predict the cumulative production of shale gas. Our framework has enough flexibility to construct models of increasing complexity that can be employed in the presence of a complex dataset or when more information is available.

Key words: gas dynamics, microfluidics, micro-/nano-fluid dynamics

\section{Introduction}

Natural gas produced from shale formations has become a significant new global energy source that is drastically altering the map of future energy supply worldwide.

$\dagger$ Email address for correspondence: ivan.lunati@unil.ch 
Although it has long been recognized that abundant gas resources are trapped in shale rocks, gas production from these formations was economically infeasible owing to the low productivity. Recently, two technological advances have been established in the industry to enable a commercially profitable production of these resources: hydraulic fracturing and horizontal drilling. It is estimated that about one-third of technically recoverable natural gas in the USA is held in shale-rock formations, and similar figures are valid on a global scale (US Energy Information Administration $2013 b$ ). In the USA, shale gas represented $39 \%$ of natural gas production in 2012, and its share is expected to grow continually in the future (US Energy Information Administration 2013a). US production growth has attracted lots of attention since shale-gas formations are also found in many parts of Europe and Asia.

As the permeability of shale is very low (of the order of a few nanodarcies), the conventional reservoir-simulation methods, which are designed for viscous-dominated displacement, cannot provide a reasonable production analysis for shale-gas reservoirs. As a result, transport phenomena in shale formations have been actively studied by many researchers with the objective of improving existing models (e.g. Roy et al. 2003; Javadpour 2009; Darabi et al. 2012; Swami \& Settari 2012). The main transport mechanisms in shale formations are viscous flow and molecular diffusion induced by gas expansion. However, as pore diameters become of the order of the molecular mean free path, no-slip boundary conditions are not appropriate and the frequent molecule-wall collisions also reduce the diffusion coefficient (Knudsen effect). Modelling shale-gas transport requires accounting for slip velocity and Knudsen diffusion in nanopores, which are normally not considered in traditional reservoir simulations. In addition to these flow processes, the analysis of gas production from shale formations is complicated by a hierarchy of structural features that contain and transport gas. Indeed, multistage hydraulic fracturing, which is employed to achieve commercially acceptable production rates, induces microseismic events that create a strongly connected network of secondary fractures in the reservoir (Zoback, Kitasei \& Copithorne 2010; Vermylen \& Zoback 2011). This complex fracture network characterized by multiple length scales plays a crucial role in determining gas production rates in the field.

In this paper we propose a simple model that allows us to estimate the production rate from fractured nanoporous shale. This model is based on the recognition that fractures contain a negligible gas volume and are much more permeable than the shale-rock matrix. As a result, the production rate is primarily controlled by the gas travel time from micro- or nanopores to the fracture system. In $\S 2$, we briefly analyse the geological structure of fractured shale formations and we discuss how the different components can be modelled. In $\S 3$, we review the physics of gas transport in shale formations, and in $\S 4$ we present a simple dual-tube model that includes all the relevant gas-transport mechanisms in an effective diffusion coefficient. In $\S 5$ we investigate the effects of pore-geometry variability, which are described by a bundle-of-dual-tubes model (BoDTM) that permits a stochastic description of the production rate and allows for great flexibility in dealing with multiporous material; and in $\S 6$ we compare the results of the BoDTM with field data from the Barnett shale-gas field. Finally, conclusions are drawn and possible extensions of the model are discussed in $\S 7$.

\section{Geological structure of fractured shale formations}

Shale is a sedimentary rock formed by compaction of silt and clay particles. Clay minerals are characterized by particle sizes that span three orders of magnitude, from a 


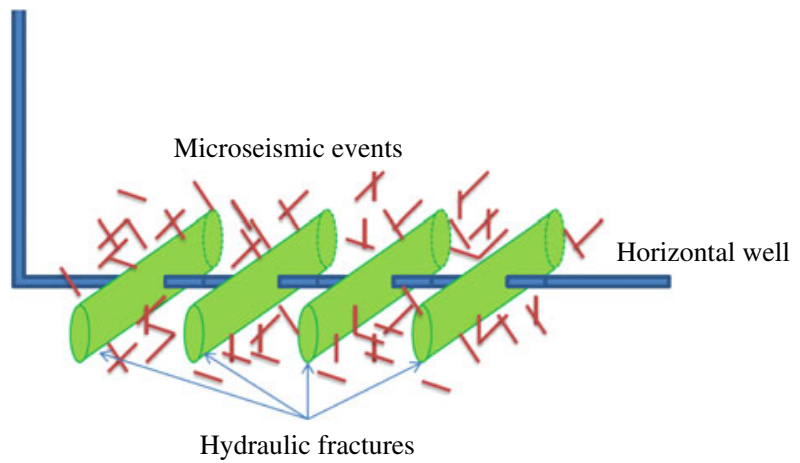

FIgURE 1. (Colour online) A schematic diagram of multistage hydraulic fracturing. Several hydraulic fractures are generated by pneumatic forcing from a horizontal well. The consequent stress release induces microseismic events that generate a network of connected microseismic fractures.

few nanometres of smectite particles to 1-20 $\mu \mathrm{m}$ of the larger dimension of kaolinite sheets (Bjørlykke 2010). After compaction, this variability in particle size produces a nanoporous material characterized by an extremely low permeability (typically of the order of a few nanodarcies, $1 \mathrm{nD} \approx 10^{-21} \mathrm{~m}^{2}$ ). To obtain an economically acceptable gas production rate, rock transport properties must be enhanced by a multistage hydraulic fracturing (Zoback et al. 2010; Vermylen \& Zoback 2011). As depicted in the schematic diagram in figure 1, several hydraulic fractures are created by injection of a high-pressure fluid into a horizontal well penetrating the shale formation longitudinally. This primary fracturing triggers a series of microseismic events that create a large number of secondary fractures. Consequently, the hydraulic fracturing generates a complex hierarchy of features that includes hydraulic fractures, a highly connected network of secondary fractures (induced or natural microfractures) and the undisturbed shale nanoporosity.

\subsection{Hydraulic fractures}

The hydraulic fractures are relatively well characterized in terms of location and geometry. For instance, a horizontal well that extends over $1500 \mathrm{~m}$ will be hydraulically fractured 10-20 times at an interval of approximately $100 \mathrm{~m}$. The fractures may extend 150-300 $\mathrm{m}$ from the well in the transverse direction (Zoback et al. 2010). The hydrofractures are extremely permeable with respect to other structural features and define the domain from which shale gas will be produced. In general, the aperture size of fractures is much smaller than the length scale of matrix block fractures, and the hydrofractures can be modelled as two-dimensional planes in an asymptotic approximation (Lough, Lee \& Kamath 1998).

\subsection{Highly connected network of secondary fractures}

The fractures induced by microseismic events are important to enhance gas productivity as they create a network that connects the hydraulic fracture planes. Figure 2 shows the image of a core sample of a shale that has been fragmented along the bedding planes and includes an extensive natural fracture cutting the core nearly vertically. The secondary fracture network breaks the shale formation into small blocks of 
(a)

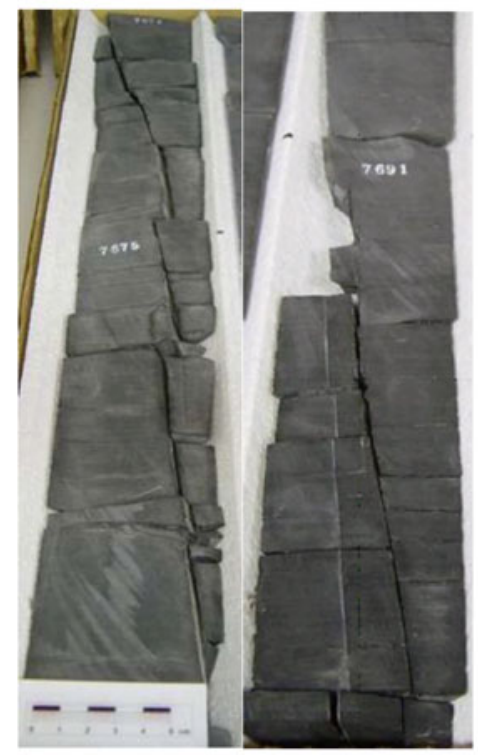

(b)

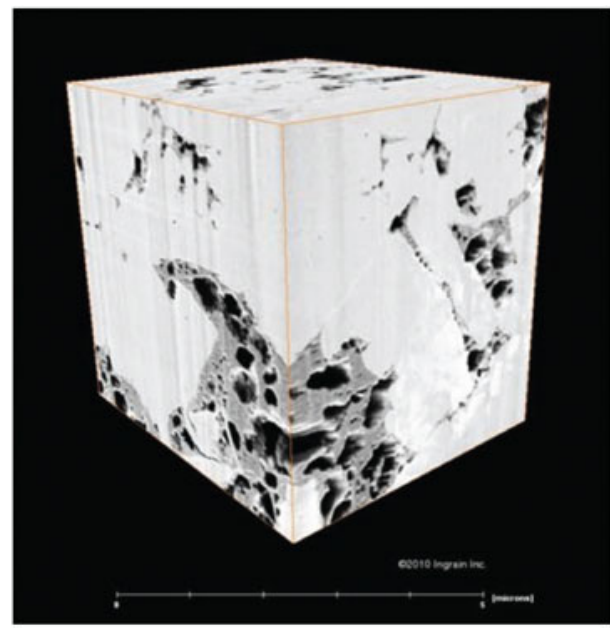

FIGURE 2. (Colour online) Illustration of the structural complexity of fractured shales. (a) Tall, planar, nearly vertical natural fractures in a core from a gas-productive shale formation; the nearly horizontal breaks are along bedding planes and were induced during coring and handling (Gale 2011). (b) X-ray imaging of micro- and nanopores in shale (Walls \& Sinclair 2011).

approximate size of half a metre. As the permeability of secondary fractures is several orders of magnitude higher than the permeability of the shale-rock matrix, the travel time in the secondary fractures is negligible with respect to the travel time in the matrix. The size of the fragmented shale blocks controls the gas production rate by determining the distance that the gas has to travel in the matrix to reach the fracture network. In field applications, full characterization of the fracture network geometry is not possible owing to rare measurements in the volume between hydraulic fracture planes, and induced microseismic fractures have to be modelled as stochastic processes (Maxwell \& Norton 2012; Sarkar et al. 2012). On the basis of the data typically available, even characterizing the fracture statistics is difficult, and one often has to rely on simplistic statistics to describe the block-size distributions.

\subsection{Disconnected fractures, induced or natural}

In addition to the connected fracture network, microseismic and natural small-scale fractures can be found within the fragmented matrix block. These fractures have a limited extension with respect to the matrix block and are disconnected from the secondary fracture network. The effects of disconnected fractures can be homogenized (Lee, Lough \& Jensen 2001) and modelled by an enhanced effective permeability that increases the transport rate from the shale-rock matrix into the fracture network.

\subsection{Micro- and nanoporosity in shales}

As the permeability is very low, measurements of transport properties are very difficult to perform and information on flow parameters is not easily available. To overcome 
(a)

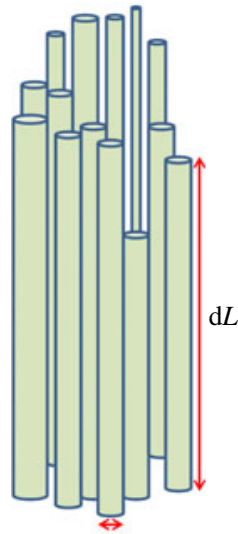

(b)

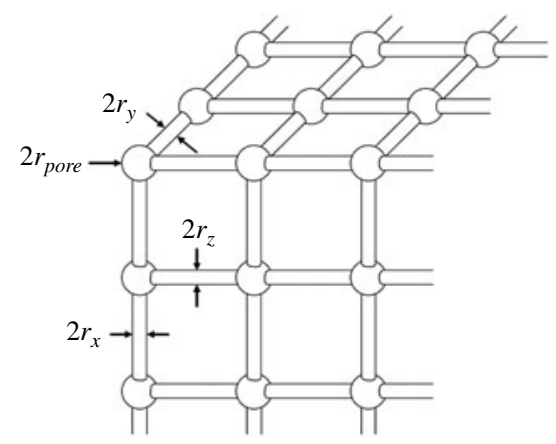

(c)

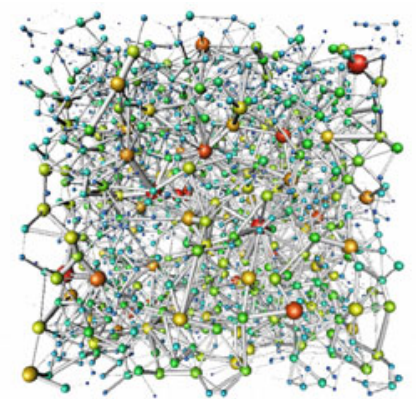

$\mathrm{d} A$

Figure 3. (Colour online) Pore network models: $(a)$ bundle-of-tubes model, $(b)$ throatbulb model and $(c)$ three-dimensional random pore network model (Dong 2007).

this lack of information, transport through the undisturbed shale porosity can be modelled by means of pore networks constructed solely on the basis of geometrical information that can be obtained from X-ray computed tomography of the porosity (an example of the complex micro- and nanopores in shales is shown in figure 2 (Walls \& Sinclair 2011)). Pore networks of different complexity (figure 3) have been extensively and successfully used to model fluid flow in porous media (Fatt 1956; Koplik 1982; Blunt \& King 1990; Lee, Padmanabhan \& Al-Sunaidi 1996). The simplest model comprises a bundle of tubes (figure 3) and despite its simplicity has proven able to predict macroscopic parameters (Fatt 1956). Throat-bulb models (figure $3 b$ ) represent an evolution of the bundle-of-tubes model (BTM) and have been employed to analyse multiphase flow in porous media (e.g. Lee et al. 1996). Recently, more complex randomly connected networks have been widely employed in the literature in the hope of better representing real pore spaces (Blunt et al. 2012).

\subsection{Structural hierarchy and bundle-of-dual-tubes model}

Modelling flow in fractured shale formations appears extremely difficult owing to the many complex structures involved. However, the problem can be greatly simplified if it is recognized that the structural features described in the previous sections form a hierarchy that goes from the most permeable (the hydraulic fractures) to the least permeable (the undisturbed shale porosity). The differences in permeability, together with the different volumes of stored gas (which are small in fractures and large in the matrix), imply that the residence time is mainly controlled by the travel time from micro- or nanopores in the undisturbed shale-rock matrix to the highly connected network of secondary fractures. As a first approximation, it can be assumed that the gas in the fracture system is instantaneously produced. This important observation allows us to focus on the description of gas flow in the shale-rock matrix (where the residence time is the longest) and to devise a simple but flexible model to estimate the production rate from a shale formation.

To describe the flow in the shale nanoporosity, we use a pore-network model. Notice that all pore networks, including complex three-dimensional random pore networks (e.g. figure $3 c$ ), are only conceptualizations of the real pore space, which 
are devised to capture relevant macroscopic effects and should be kept as simple as possible. Ultimately, the complexity of the network model to be employed depends on the complexity of the physical phenomena to be analysed. Since we are interested in single-phase flow of gas, a detailed description of the complex geometry and connectivity as in three-dimensional pore networks is not necessary and the models can be greatly simplified.

Conceptually, the flow field can be decomposed into a bundle of pathways (or streamtubes) that consist of successions of many pores and throats. Owing to their large number, the effects of pores and throats can be homogenized and each pathway is described by two effective diameters: a larger storage diameter, which describes the effective average capacity of the pores along the pathway, and a smaller diameter, which describes the effective average permeability of the throats along the pathways. (Notice that the pore network can also be conceptually reduced to a tube characterized by two effective diameters also applying a renormalization procedure to a network of resistances and capacitors.) To account for the effects of possible long correlation in pore or throat size, we allow different pathways to have different effective diameters and we therefore consider a bundle of tubes with statistical distribution of the parameters.

This conceptual model leads to a BoDTM, which is a generalization of classic BTMs. BTMs have a long tradition in describing flow through porous media and, despite their simplicity, they have proven able to effectively predict macroscopic parameters since the pioneering work of Fatt (1956). To account for the fact that most of the matrix storage volume is provided by pores connected by throats, the model comprises a bundle of dual tubes, each of which consists of two tubes of different diameter: the smaller tube represents the throat size in dynamic flow calculations, whereas the larger tube represents the gas capacity from bulbs and throats. The use of a bundle of tubes allows accounting for pore and throat size variabilities in the shale-rock matrix.

The effect of the secondary fracture network on the production rate is to significantly reduce the distance that the gas has to travel in the matrix, which is determined by the typical size of the fragmented shale blocks. Therefore, the dual-tube length in the BoDTM depends on the statistical distribution of the block size and on the tortuosity of the pore space. This simple model offers enough flexibility to estimate the production rate from fractured shale formations.

\section{Physics of gas transport in shale formations}

\subsection{Mass balance and transport mechanisms}

A general mass-balance equation can be written in the form

$$
\frac{\partial}{\partial t}(\varphi \rho)+\nabla \cdot \boldsymbol{j}=q,
$$

where $\rho$ is the density of the gas, $\varphi$ is the accessible storage volume fraction, $\boldsymbol{j}$ is the mass flux and $q$ is a source term representing physicochemical reactions. In general, the flux term depends on the spatial distribution of the density and should be considered a functional, $\boldsymbol{j}[\rho(\boldsymbol{x})]$. The flux term can be approximated by a Taylor expansion, which can be truncated by invoking locality. Retaining the first two terms in the expansion, the mass-flux component in the $i$ direction is

$$
j_{i}=u_{i} \rho-D_{i j} \frac{\partial \rho}{\partial x_{j}},
$$


where the first term represents the advective flux due to the mean velocity $u_{i}$, whereas the second term describes the net effects of velocity fluctuations and is related to the density gradient by the second-rank tensor $D_{i j}$.

The particular form of the coefficients in (3.2) depends on the dominating physical processes. For flow in a porous formation, it can be assumed that pressure forces are instantaneously balanced by the viscous drag exerted by the solid matrix, and the mean gas velocity can be described by Darcy's law,

$$
u_{i}=-\frac{k_{i j}}{\mu} \frac{\partial p}{\partial x_{j}},
$$

where $\mu$ is the viscosity of the gas and $k_{i j}$ is the absolute permeability tensor, which is a characteristic of the formation and does not depend on fluid properties.

The coefficient of the second term in (3.2) describes the net effects of velocity fluctuations. For our purposes, it is sufficient to consider microscopic fluctuations due to thermal agitation of the molecules, which can be described by a tensor of the form

$$
D_{i j}=D \varphi \theta_{i j},
$$

where $D$ is the diffusion coefficient in the bulk fluid, which is isotropic, and $\theta_{i j}$ is a tensor proportional to the square of the inverse of the tortuosity (Bear 1972; Epstein 1989). In our BoDTM, mechanical dispersion resulting from velocity fluctuations among pores are described by the variable properties assigned to each dual tube of the bundle.

In the rest of this section, we briefly review the relevant gas-transport processes in shale formations and highlight the results that will be used to derive the governing equations of the BoDTM.

\subsection{Molecular diffusion coefficient}

The viscosity and the molecular diffusion coefficient describe the ability of molecular collisions to transfer momentum and mass, respectively. They are proportional to the mean free path, $\ell$, and to the thermal velocity, $v_{T}$ (Hirschfelder, Curtiss \& Bird 1954; Poling, Prausnitz \& O'Connell 2000; Welty et al. 2007). Using elementary kinetic theory and assuming a Maxwell-Boltzmann distribution of the velocity, the mean free path becomes

$$
\ell=\left(\frac{m}{\sqrt{2} \sigma}\right) \frac{1}{\rho} .
$$

Some typical values under reservoir conditions are shown in table 1 . The thermal velocity, interpreted as the mean magnitude of the molecular velocity, is given by

$$
v_{T}=\sqrt{\frac{8 k_{B}}{\pi m}} T^{\frac{1}{2}},
$$

where $\sigma$ is the cross-sectional area of collision, $k_{B}=1.38 \times 10^{-23} \mathrm{~J} \mathrm{~K}^{-1}$ is the Boltzmann constant and $m$ is the molecular mass (i.e. the mass of a single molecule). Then, we have the molecular diffusion coefficient

$$
D^{m}=\frac{1}{3} \ell v_{T}=\xi_{D} \frac{\sqrt{k_{B} m / \pi}}{\sigma} \frac{T^{\frac{1}{2}}}{\rho},
$$

where the numerical prefactor is $\xi_{D}=\frac{2}{3}$. 


$\begin{array}{lccc}T\left({ }^{\circ} \mathrm{C}\right) & p(\mathrm{MPa}) & \rho\left(\mathrm{kg} \mathrm{m}^{-3}\right) & \ell(\mathrm{nm}) \\ 100 & 1 & 5.2 & 8 \\ 100 & 10 & 54 & 0.8 \\ 150 & 10 & 46 & 0.9 \\ 150 & 30 & 125 & 0.3\end{array}$

TABLE 1. Some values of the mean free path at different reservoir conditions.

More refined models based on a first approximation of the Boltzmann equation yield the same result but with $\xi_{D}=\left(\frac{5}{16}\right) \pi$ and a temperature-dependent cross-sectional area, $\sigma(T)$, if an intermolecular potential is considered (Chapman \& Cowling 1939; Hirschfelder et al. 1954). For simplicity, we neglect the dependence of $\sigma$ on $T$, and write the dependence of the diffusion coefficient on temperature and density as

$$
D^{m}=\left(\frac{\rho_{0}}{\rho}\right)\left(\frac{T}{T_{0}}\right)^{\frac{1}{2}} D_{0}^{m},
$$

where $D_{0}^{m}$ is the value at some reference conditions $\left(p_{0}, T_{0}, \rho_{0}\right)$, which we take as the initial conditions in the formation. The equations above, which we have presented for a pure gas and self-diffusion, are sufficient for our purposes. Analogous relationships among macroscopic quantities for gas mixtures can be derived by considering binary diffusion in addition to self-diffusion. In that case, the mass has to be interpreted as the harmonic mean of the molecular masses and $\sigma$ is the appropriate cross-sectional area of collisions (details can be found in Curtiss \& Hirschfelder (1949) and Hirschfelder et al. (1954)).

\subsection{Diffusion in nanopores and effective diffusion coefficient}

When the gas is confined in a pore whose diameter, $d$, is comparable to or smaller than $\ell$, interactions with the solid wall are more likely than intermolecular interactions and dominate. In this case, we replace $\ell$ by $d$ in (3.7), which yields the Knudsen diffusion coefficient

$$
D^{k}=\frac{1}{3} d v_{T}=D^{m} \frac{d}{\ell}=D^{m} \frac{d}{\ell_{0}} \frac{\rho}{\rho_{0}}=\frac{D^{m}}{K n} \frac{\rho}{\rho_{0}},
$$

where the Knudsen number,

$$
K n=\frac{\ell_{0}}{d}
$$

defines the ratio of Knudsen diffusion to standard diffusion at reference conditions.

Methane, for instance, has molecular mass $m=16 \mathrm{Da}=2.66 \times 10^{-26} \mathrm{~kg}$, normal vapour density $\rho_{N}=0.72 \mathrm{~kg} \mathrm{~m}^{-3}$, and approximate cross-sectional area $\sigma=\pi \delta^{2}=$ $0.45 \mathrm{~nm}^{2}$, where $\delta \sim 3.8 \AA$ is the molecular diameter. From (3.5) we can calculate the mean free path at standard conditions as $\ell \approx 60 \mathrm{~nm}$, which yields $K n=60$ in a nanopore and $K n=0.06$ in a micropore. With a reservoir pressure of $10 \mathrm{MPa}$ and a temperature of $150{ }^{\circ} \mathrm{C}$, the density is $46 \mathrm{~kg} \mathrm{~m}^{-3}$ and the mean free path $\ell \approx 0.9 \mathrm{~nm}$, which yields $K n=0.9$ in a nanopore and $K n=9 \times 10^{-4}$ in a micropore. This simple estimate shows that the Knudsen diffusion can be important in the diffusion process 
in a nanopore; at a larger density, however, the effects of Knudsen diffusion become less important because collisions between molecules become more likely.

Since both molecular diffusion and Knudsen diffusion can be important, we introduce a total diffusion coefficient,

$$
D=D^{m}\left[1+\left(K n \rho_{0} / \rho\right)^{1 / \gamma}\right]^{-\gamma},
$$

which describes their combined effects and tends to $D^{m}$ when $\left(K n \rho_{0} / \rho\right) \ll 1$ and to $D^{k}$ when $\left(K n \rho_{0} / \rho\right) \gg 1$. The averaging parameter $\gamma$ must be positive to preserve the fact that the simultaneous effects of molecular and wall collisions lead to a smaller diffusion coefficient. In the following we will use $\gamma=1$, which leads to the Bosanquet equation.

\subsection{Viscosity}

As the viscosity and the molecular diffusion coefficient both originate from microscopic velocity fluctuations, they are closely connected and related through the dimensionless Schmidt number,

$$
S c=\frac{\mu}{\rho D^{m}} .
$$

From the elementary kinetic theory we have

$$
\mu=\frac{1}{3} \rho \ell v_{T}=\xi_{\mu} \frac{\sqrt{k_{B} m / \pi}}{\sigma} T^{\frac{1}{2}},
$$

with $\xi_{\mu}=\frac{2}{3}$, which implies that the viscosity depends only on temperature and not on density. By substituting (3.7) and (3.13) into (3.12), we have $S c=1$. Again, more refined models yield a similar result, but the numerical prefactors, $\xi_{D}$ and $\xi_{\mu}$, and the cross-sectional areas, $\sigma$, are different for the viscosity and the diffusion coefficient, which leads to Schmidt numbers that are different from unity. For a first-order approximation of the Boltzmann equation we have $\xi_{\mu}=\frac{3}{8} \pi$, and $S c$ can depend on temperature if an intermolecular potential is considered (Chapman \& Cowling 1939; Hirschfelder et al. 1954). Assuming that the cross-sectional areas for viscosity and diffusion coefficient are approximately the same, we have $S c=\frac{6}{5}$, which is close to unity.

If we neglect the dependence of the cross-sectional area on the temperature, we can write

$$
\mu=\mu_{0}\left(\frac{T}{T_{0}}\right)^{\frac{1}{2}},
$$

where $\mu_{0}$ is the viscosity measured at reference temperature $T_{0}$.

\subsection{Effects of slip velocity in nanopores}

For a cylindrical tube or a fracture, the permeability in the longitudinal direction can be calculate from the Hagen-Poiseuille equation as

$$
k=\kappa d^{2},
$$

where $d$ denotes the diameter of a circular tube or the aperture of a plane fracture, and the dimensionless constant is $\kappa=\frac{1}{32}$ for a circular tube and $\kappa=\frac{1}{12}$ for a plane 
fracture, respectively. Equation (3.15) shows that the permeability rapidly decreases with the pore size; however, for very small pores of size comparable to the mean free path, $\ell$, the no-slip condition at the solid boundary breaks down and the equation has to be modified (Brown et al. 1946; Roy et al. 2003; Cui, Bustin \& Bustin 2009).

Based on results from the kinetic theory of monatomic gases (Maxwell 1879), Brown et al. (1946) neglected thermal creep and proposed to write the longitudinal permeability in a tube corrected to account for slippage effects as

$$
k=\left[1+4 \frac{\ell}{d}\left(\frac{2}{\sigma_{V}}-1\right)\right] \kappa d^{2},
$$

where $\kappa=\frac{1}{32}$ and $0 \leqslant \sigma_{V} \leqslant 1$ is the tangential-momentum accommodation coefficient, which indicates the fraction of molecules that are diffusively reflected by the wall. We assume $\sigma_{V}=1$, which corresponds to a rough surface that reflects all molecules diffusively (Roy et al. 2003). Using the definition of Knudsen number, (3.10), we write

$$
k=\left[1+4\left(K n \rho_{0} / \rho\right)\right] \kappa d^{2} .
$$

Owing to the second term in brackets, the permeability in pores of small size, comparable with $\ell$, becomes linear, rather than quadratic, in $d$.

\subsection{Equation of state}

Temperature, pressure and density are related by the equation of state, which can be written as

$$
Z=\frac{m p}{\rho k_{B} T},
$$

where $Z$ is the compressibility factor. For an ideal gas $Z=1$, whereas for a real gas we have $Z=Z(\rho, T)$ (rigorously, the compressibility factor of a gas mixture should depend also on the partial densities of the gas components (Poling et al. 2000)). The equation of state allows one of the variables, e.g. the pressure, to be eliminated, but requires an energy equation to be solved to determine the temperature evolution.

In the case of flow in shale formations, the low porosity of the rock matrix and the small gas flux suggest that the dynamics of the gas does not modify the temperature, which is controlled by the large thermal capacity and the large thermal conductivity of the rock. We therefore assume that the process is isothermal and we write the equation of state in the form

$$
\frac{p}{p_{0}}=\left[\frac{Z\left(\rho, T_{0}\right)}{Z_{0}}\right] \frac{\rho}{\rho_{0}},
$$

where $Z_{0}=Z\left(\rho_{0}, T_{0}\right)$. This allows us to relate pressure and density increments,

$$
\zeta(\rho)=\frac{\rho_{0}}{p_{0}}\left(\frac{\partial p}{\partial \rho}\right)_{T_{0}}=\frac{Z\left(\rho, T_{0}\right)}{Z_{0}}\left[1+\frac{\rho}{Z}\left(\frac{\partial Z}{\partial \rho}\right)_{T_{0}}\right] \approx 1,
$$

where the last approximation is valid if $Z$ varies sufficiently slowly with $\rho$ (in particular, it is exact for an ideal gas). 
(a)

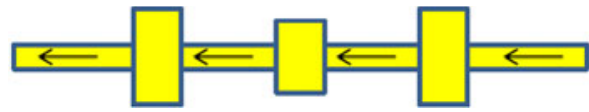

(b)

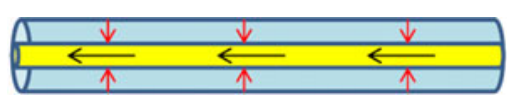

FIGURE 4. (Colour online) One-dimensional tube models representing a gas pathway (or streamtube): $(a)$ one-dimensional throat-bulb model; $(b)$ one-dimensional dual-tube model. A series of pores and throats in series are described by a tube characterized by two distinct diameters: a larger storage diameter (darker grey; light blue online), which describes the average storage capacity along the pathway; and a smaller diameter (lighter grey; yellow online) that describes the average permeability along the pathway.

\subsection{Adsorption/desorption process and organic material}

As pores in shale formations are small, the amount of gas adsorbed on the solid surfaces is in general not negligible. Organic material (kerogen) is often identified in shale and can adsorb important quantities of gas that desorb back into the porosity when the pressure is lowered. At high pressure, however, the amount of adsorbed gas reaches a saturation level. The excessive Langmuir absorption is maximum at 20 bar and then decreases quickly when the pressure increases to a few hundred bars (Meyers \& Monson 2002). This implies that the contribution of the desorbed gas to production is particularly relevant below 20 bar, but less important at higher pressure. Based on transport rate analysis (Leahy-Dios et al. 2011), the amount of gas desorbed from kerogen is relatively small compared to free gas trapped in the rock pores. For simplicity, we do not include the Langmuir adsorption/desorption in the following analysis. When this process is relevant (e.g. in methane production from a coal-bed formation), this can be easily included in the model by making the storage volume a nonlinear function of pressure, which allows the modelling of the Langmuir adsorption, as well as the effects of geomechanical deformation of porosity. Except in extremely small pores (very large $K n$ ), the adsorbed layer has no noticeable impact on the diameter of the tube and the modified permeability in (3.17) can be used. Also, the slippage correction to the permeability, which assumes diffusive reflection, is again justified owing to the fact that wall roughness is increased by adsorption.

\section{The dual-tube model}

To simplify the gas-flow equations, each tube is assumed straight and tortuosity is represented by the tube length. An important feature of our conceptual model is that, in order to account for the fact that transport pathways comprise a sequence of bulbs and throats, we consider dual tubes, which are characterized by two different diameters, $d_{S}$ and $d$, which represent an effective storage diameter and an effective conductive diameter, respectively (figure 4).

\subsection{Governing equation}

First, we consider a tube of length $L$ that initially contains gas at density $\rho_{0}$. At time zero, the two ends of the tube are put in contact with a secondary fracture where the gas has density $\rho_{f}$. Taking advantage of the symmetry of the problem, we consider only a half-tube of size $L / 2$. (If only one end of the tube is connected to a fracture network, the whole length of the tube will be used to calculate the gas transport.) We write the transversely integrated gas-balance equation as (a detailed derivation of this 
equation in given in appendix A)

$$
\frac{1}{\omega} \frac{\partial \rho}{\partial t}=\frac{\partial}{\partial x}\left\{\left[\operatorname{Pe} \zeta(\rho)\left(\frac{\rho}{\rho_{0}}+4 K n\right)+\frac{1}{\left(\rho / \rho_{0}\right)+K n}\right] D_{0}^{m} \frac{\partial \rho}{\partial x}\right\},
$$

with initial and boundary conditions

$$
\begin{aligned}
& \rho(x, t=0)=\rho_{0}, \\
& \rho(x=0, t)=\rho_{f}, \\
& \left.\frac{\partial \rho}{\partial x}\right|_{(x=L / 2, t)}=0,
\end{aligned}
$$

and where we have defined the dimensionless numbers

$$
\omega=\frac{d^{2}}{d_{S}^{2}}=\left(\frac{\pi}{4} \frac{\ell_{0}^{2} L}{V_{S}}\right) \frac{1}{K n^{2}} \quad \text { and } \quad P e=\frac{\kappa d^{2} p_{0}}{D_{0}^{m} \mu_{0}} .
$$

The former is the ratio between the storage volume, $V_{S}=\pi d_{S}^{2} L / 4$, and the conductive volume, $V_{C}=\pi d^{2} L / 4$, and can be regarded as the conductive fraction of the porosity. The latter is the Péclet number, which compares advection to diffusion. By recalling the results of kinetic theory, we write

$$
P e=P e_{*} K n^{-2},
$$

where the reduced Péclet number, $P e_{*}=(\pi / 2)\left(Z_{0} \kappa / \xi_{\mu} \xi_{D}\right)$, depends on the specific kinetic model that describes the fluid, $\xi_{D}$ and $\xi_{\mu}$, on the compressibility factor at reference condition, $Z_{0}$, and on the geometric factor, $\kappa$ (see appendix B for details).

In (4.1), the quantity in square brackets is a correction to the diffusion coefficient that describes all relevant transport mechanisms: Darcy flow, the effects of slip velocity, molecular diffusion and Knudsen diffusion. Molecular diffusion and Knudsen diffusion are averaged harmonically, which leads to a dominance of the smaller coefficient: the combined effects of collisions between molecules and collisions with the solid reduce the effective mean free path, thus lowering the diffusion coefficient. Employing the elementary kinetic theory and assuming $Z_{0} \approx 1$, the reduced Péclet number becomes proportional to $\kappa$ and can be regarded as a modified geometric factor, which is $P e_{*}=\frac{1}{9}$ for a straight tube (appendix B).

\subsection{Dimensionless equation and effective diffusion coefficient}

By introducing the dimensionless variables,

$$
\rho^{\prime}=\frac{\rho}{\rho_{0}}, \quad x^{\prime}=\frac{x}{L} \quad \text { and } \quad t^{\prime}=\frac{t}{t_{c}}=\frac{\omega}{K n} \frac{D_{0}^{m}}{L^{2}} t,
$$

and after some algebraic manipulations (see appendix A for details), the gas-balance equation is recast in a simple dimensionless form,

$$
\frac{\partial \rho^{\prime}}{\partial t^{\prime}}=\frac{\partial}{\partial x^{\prime}}\left[D^{e}\left(\rho^{\prime} / K n, P e_{*}\right) \frac{\partial \rho^{\prime}}{\partial x^{\prime}}\right]
$$




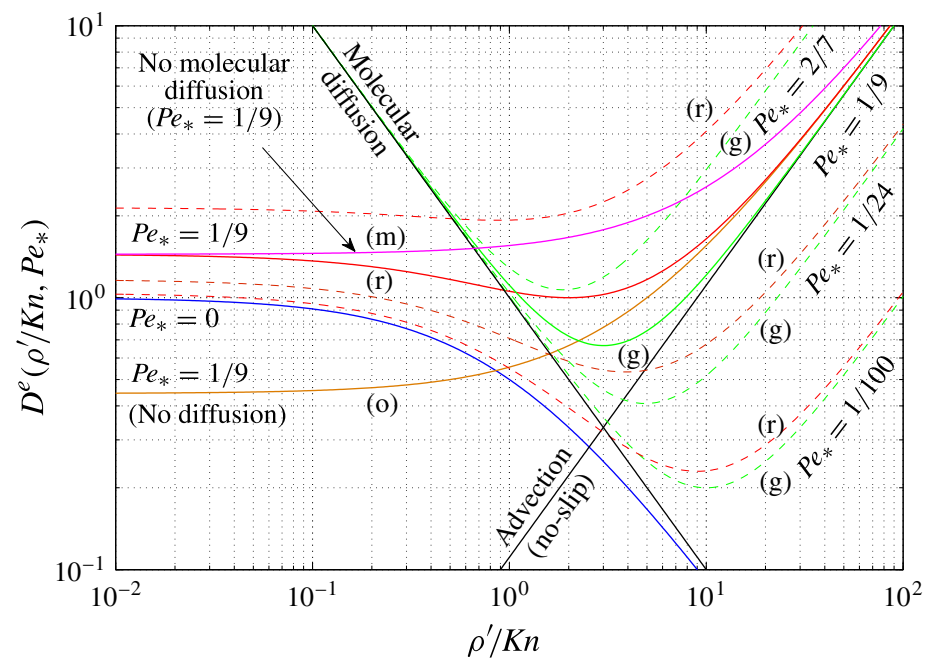

FIGURE 5. (Colour online) The dimensionless effective diffusion coefficient, $D^{e}$, as a function of the dimensionless density $\rho^{\prime} / K n=\rho \sqrt{2} \sigma d / m$ and for different $P e_{*}$. The contributions of the different transport mechanisms are shown for $P e_{*}=\frac{1}{9}$ (solid red (r) line): advection including slippage effects, $P e_{*}\left(\rho^{\prime} / K n+4\right)$ (orange (o)); molecular and Knudsen diffusion, $\left(\rho^{\prime} / K n+1\right)^{-1}$ (blue (b)); advection without slippage effects and molecular diffusion, $P e_{*} \rho^{\prime} / K n+K n / \rho^{\prime}$ (green $(\mathrm{g})$ ); and advection with slippage and Knudsen diffusion, $P e_{*}\left(\rho^{\prime} / K n+4\right)+1$ (magenta $\left.(\mathrm{m})\right)$. The two straight black lines represent the effects of standard molecular diffusion and advection (with no slip). Finally, the dashed lines plot the effective diffusion coefficient (red (r)) and the effective diffusion coefficient without Knudsen diffusion and slippage (green $(\mathrm{g})$ ) for three other values of $\mathrm{Pe}_{*}(2 / 7,1 / 24$ and $1 / 100)$.

with initial and boundary conditions

$$
\begin{gathered}
\rho^{\prime}\left(x^{\prime}, t^{\prime}=0\right)=1, \\
\rho^{\prime}\left(x^{\prime}=0, t^{\prime}\right)=\rho_{f} / \rho_{0}, \\
\left.\frac{\partial \rho^{\prime}}{\partial x^{\prime}}\right|_{\left(x^{\prime}=\frac{1}{2}, t^{\prime}\right)}=0 .
\end{gathered}
$$

The effective diffusion coefficient,

$$
D^{e}\left(\rho^{\prime \prime}, P e_{*}\right)=P e_{*}\left(\rho^{\prime \prime}+4\right)+\frac{1}{\rho^{\prime \prime}+1},
$$

depends only on $P e_{*}$ and it is plotted as a function of $\rho^{\prime \prime}=\rho^{\prime} / K n=\rho \sqrt{2} \sigma d / m$ in figure 5, together with the contributions of different transport mechanisms. (Notice that the dimensional diffusion coefficient is obtained by multiplying the effective diffusion coefficient by $D_{0}^{m} / K n$, as shown in appendix A.)

Increasing the tube diameter or the gas density has the same effect: in both cases the ratio of molecular collisions to wall collisions increases. At high density and small Knudsen numbers, standard advection (with no-slip) dominates; whereas slippage effects and Knudsen diffusion prevail at lower density and large $K n$. Slippage effects 

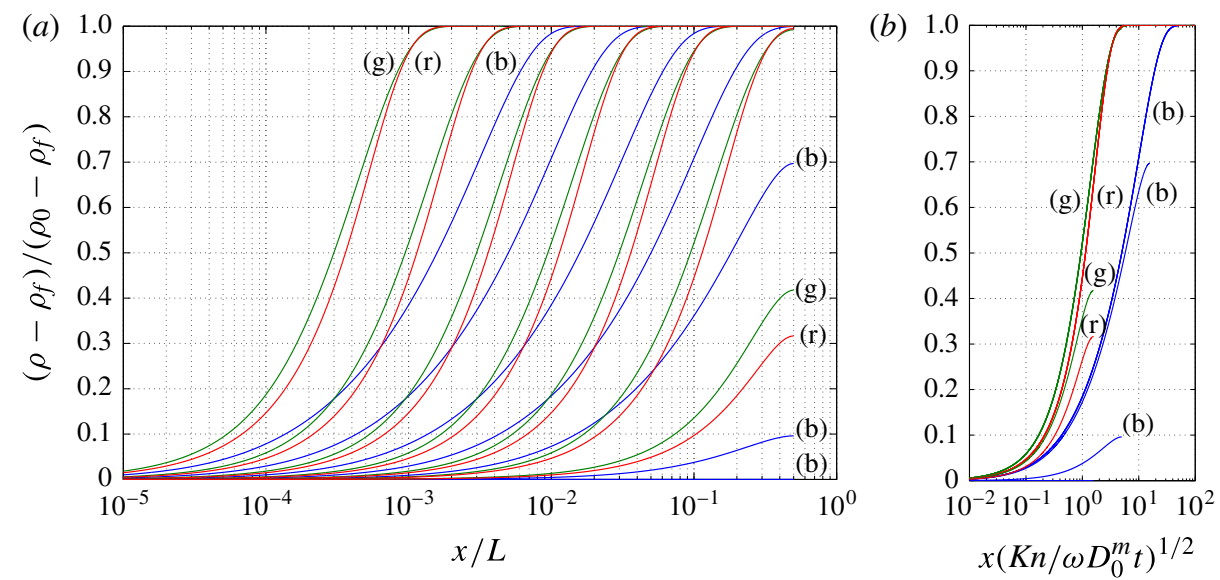

FIgURE 6. (Colour online) Density profile at different dimensionless times $t^{\prime}=$ $\omega D_{0}^{m} t / K n L^{2}=10^{-7}, 10^{-6}, 10^{-5}, 10^{-4}, 10^{-3}, 10^{-2}, 10^{-1}$. Shown are the results for three different Knudsen numbers $\left(K n=10^{-3}\right.$, in blue (b); $K n=10^{-1}$, in green (g); and $K n=10$, in red $(\mathrm{r})$ ) and $\rho_{f} / \rho_{0}=0.1$. (Notice that the blue curve corresponding to $K n=10^{-3}$ and $t^{\prime}=10^{-1}$ is close to zero and overlaps with the horizontal axis.) Before the density starts decreasing at the right boundary $\left(x / L=\frac{1}{2}\right)$, the solution is approximately self-similar $(a)$, and the profiles collapse on a single profile by the scaling $x\left(K n / \omega D_{0}^{m} t\right)^{\frac{1}{2}}(b)$. The dimensionless density $\left(\rho / \rho_{0}\right)$ at the right boundary is below 0.99 at $t^{\prime}=1.8 \times 10^{-4}, 1.1 \times 10^{-2}$ and $1.2 \times 10^{-2}$ for $K n=10^{-3}, 10^{-1}$ and 10 , respectively.

are significant at relatively large values of $\rho^{\prime} / K n$ (under our hypothesis of rough walls, $\sigma_{v}=1$ in (3.16)). Molecular diffusion is important at intermediate values and contributes to creating a diffusivity minimum at $\rho^{\prime} / K n \approx 2$. This minimum is due to our model for total diffusion (4.12); physically, it originates because, at intermediate density and tube sizes, both molecule-molecule and molecule-wall interactions are important, increasing the number of collisions and reducing the mean free path.

\subsection{Density profile}

Equation (4.8) is a generalized porous media equation with diffusion coefficient that is not a simple power-law function. In this case, self-similar solutions cannot perform an arbitrary scaling and they exist only for particular initial and boundary conditions (see e.g. Vázquez 2007). The effective diffusion coefficient in (4.12) can be approximated by a power law in several limiting cases (e.g. negligible molecular diffusion, large $P e_{*}$ and very high or very low densities). However, owing to the fact that we are interested in solutions in a finite domain, we compute the density profile numerically.

The time evolution of the density profiles is shown in figure 6 for three different Knudsen numbers $(K n=0.001,0.1$ and 10). The sudden pressure drop at the left boundary causes a gas expansion with a density decrease that propagates to the centre of the tube (the no-flow boundary condition at $L / 2$ ). As long as the density at the right boundary $\left(x^{\prime}=\frac{1}{2}\right)$ does not appreciably decrease, the solution can be approximated by the solution in an infinite domain, which admits a self-similar solution of the form $\rho^{\prime}\left(x^{\prime}, t^{\prime}\right)=\tilde{\rho}\left(x^{\prime} / t^{\prime \frac{1}{2}}\right)$ (see e.g. Vázquez 2007). Figure 6 shows that the solution at earlier time is approximately self-similar and, for a given $K n$, the profiles collapse to a single curve by appropriate scaling. 

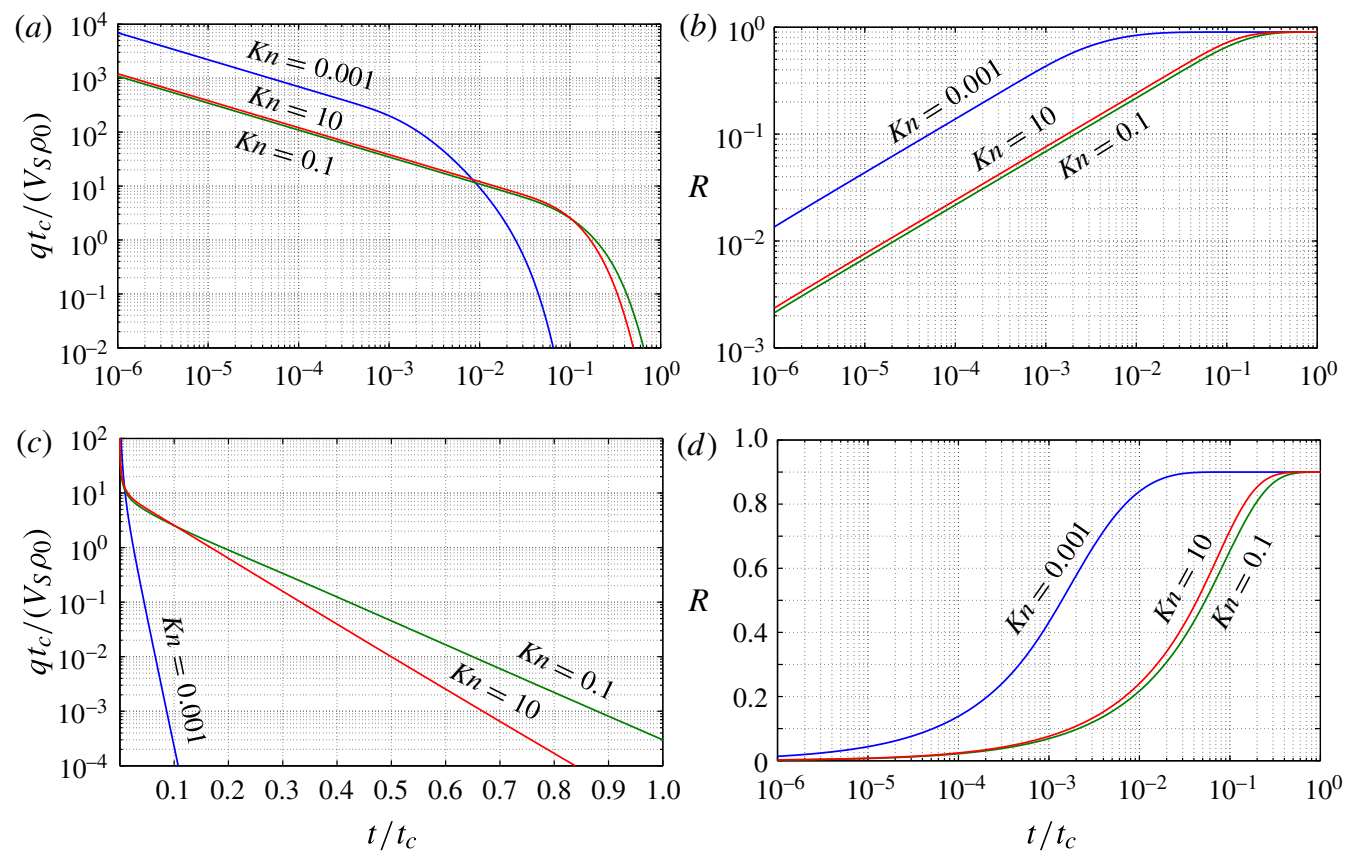

FIGURE 7. (Colour online) Dimensionless production rate, $q t_{c} /\left(V_{S} \rho_{0}\right)$, and recovery fraction, $R$, as functions of the dimensionless time $t / t_{c}\left(t_{c}=K n L^{2} / \omega D_{0}^{m}\right)$ for three Knudsen numbers $\left(K n=10^{-3}\right.$, in blue; $K n=10^{-1}$, in green; and $K n=10$, in red): $(a, b) \log -\log$ plots; $(c, d)$ semi-log plots.

\subsection{Production rate and recovery}

The gas production rate is calculated from the gradient of the density profile at the left boundary of the tube $\left(x^{\prime}=0\right)$, i.e.

$$
q_{K n}^{\prime}\left(t^{\prime}\right)=2 D^{e}\left(\rho_{f} /\left(\rho_{0} K n\right), P e_{*}\right)\left[\frac{\partial \rho^{\prime}}{\partial x^{\prime}}\left(x^{\prime}, t^{\prime}\right)\right]_{x^{\prime}=0},
$$

and it is plotted as a function of the dimensionless time in figure 7. (The factor 2 appears because the solution of (4.8)-(4.11) only describes half of the tube; gas is also produced at the other tube end, $x^{\prime}=1$.) At earlier time, the solution is approximately self-similar and the production rate is inversely proportional to the square root of time, i.e. $q^{\prime} \sim 1 / \sqrt{t^{\prime}}$. At later time, the presence of the symmetry boundary influences the solution. After a transition period, the production rate declines exponentially. The dimensionless production rates for $K n=0.1$ and $K n=10$ are very similar owing to the relatively flat shape of the effective diffusion for the corresponding values of $\rho^{\prime} / K n$, i.e. $1<\rho^{\prime} / K n<10$ and $0.01<\rho^{\prime} / K n<0.1$, respectively. However, the dimensional production rate,

$$
q(t)=\frac{V_{S} \rho_{0}}{t_{c}} q_{K n}^{\prime}\left(t / t_{c}\right),
$$

is very different because $t_{c}=(K n / \omega)\left(L^{2} / D_{0}^{m}\right)$ depends on $K n$.

To facilitate comparison, we consider the recovery fraction

$$
R(t)=\frac{1}{V_{S} \rho_{0}} \int_{0}^{t} q(\tau) \mathrm{d} \tau=\int_{0}^{t / t_{c}} q_{K n}^{\prime}\left(\tau^{\prime}\right) \mathrm{d} \tau^{\prime},
$$


which is the fraction of the gas initially present in the formation that has been recovered at time $t$. Figure 7 shows that $40 \%$ of the gas is recovered at $t^{\prime} \approx 8 \times 10^{-4}$ for $K n=10^{-3}$, and at $t^{\prime} \approx 3 \times 10^{-2}$ for both $K n=0.1$ and $K n=10$.

Since the characteristic time $t_{c}$ is different in the three cases, this $40 \%$ recovery is reached at very different physical times. Assuming, for instance, that length and storage volume are the same in the three cases, the characteristic time is $t_{c} \sim K^{3}$. This means that $40 \%$ of the gas is recovered about seven orders of magnitude faster for $K n=10^{-3}$ than for $K n=0.1$ (the ratio between the $40 \%$ recovery times is $2.5 \times 10^{-7}$ ); also, for $K n=10$ the recovery is six orders of magnitude slower than for $K n=0.1$ (the ratio between the $40 \%$ recovery times is $10^{6}$ ). If the storage diameter is proportional to the conductive diameter (i.e. $\omega$ is constant), we have $t_{c} \sim K n$ and the differences in time to reach $40 \%$ recovery are reduced to four and two orders of magnitude, respectively. Notice, however, that in the second case the volumes are different in the different tubes and the ratio between the production rates is again of eight and six orders of magnitude. Owing to the form of the effective diffusion coefficient in figure 5, this difference in production rate is smaller than what it is expected for an advection-dominated process, in which $q \sim K^{-4}$ based on Poiseuille flow.

\section{The bundle-of-dual-tubes model}

In this section we show how the production rate from a dual capillary tube can be used to estimate the production rate from a fractured nanoporous shale formation. Because of high contrasts in permeability and storage capacity between the fractures and the nanoporosity, we assume that the gas in the fracture network is produced instantaneously. Then, the production rate can be approximated by the flux from the porous matrix blocks into the fracture network. To account for the variability of gas pathways in the nanoporous shale, we employ a BoDTM, whose properties depend on the pore geometry of the formation. In the following, we investigate the effects of pore-space variability on recovery and production rate.

\subsection{Geometric characterization of the reservoir}

We denote by $V_{R}$ the volume of formation embedded by two hydraulic fractures which has porosity $\varphi$. The two hydraulic fractures are connected by a network of secondary fractures and determine the region from which gas is produced. As the secondary fractures have smaller storage capacity and higher conductivity than the shale matrix, the production rate from the volume $V_{R}$ can be approximated by the flow rate from the shale-matrix nanoporosity into the secondary fracture network. Under this assumption, the geometry of the secondary fracture network only affects the length of the tubes to be used in the BoDTM, which is $L=\theta L_{B}$, where $L_{B}$ is the size of the blocks into which the shale matrix is subdivided by the fracture network, and $\theta$ is the tortuosity.

The pore geometry of the shale matrix determines the conductive diameters, $d$, and the storage volumes, $V_{S}$, of the tube bundle. The statistics of the tube bundle is fully described by a joint probability density function (p.d.f.) $f_{d, L, V_{S}}\left(d, L, V_{S}\right)$, or equivalently by

$$
f_{K n, t_{c}, V_{S}}\left(K n, t_{c}, V_{S}\right),
$$

which is the joint p.d.f. of the Knudsen numbers, $K n=\ell_{0} / d$, the characteristic times, $t_{c}=K n^{3}\left(V_{S} L /\left(\pi \ell_{0}^{2} D_{0}^{m} / 4\right)\right)$, and the storage volumes, $V_{S}$, of the tubes. In practical applications the joint statistics should be inferred from data (e.g. from computed tomography images), but, owing to limited information, it might be necessary to postulate it on the basis of some a priori knowledge or hypothesis. 


\subsection{Production rate and recovery from a bundle of tubes}

If the joint probability is known, the total production rate of the bundle can be computed as

$$
Q(t)=N\langle q(t)\rangle
$$

where

$$
\langle *\rangle=\iiint * f_{K n, t_{c}, V_{S}}\left(K n, t_{c}, V_{S}\right) \mathrm{d} K n \mathrm{~d} t_{c} \mathrm{~d} V_{S}
$$

is the average operator, and $N$ is the number of tubes in the bundle, which is determined by the volume constraint

$$
N\left\langle V_{S}\right\rangle=\varphi V_{R}
$$

Using the dimensionless solution for a dual tube, (4.13), which depends on $K n=$ $\ell_{0} / d$ (for a tube and using elementary kinetic theory, we have $P e_{*}=\frac{1}{9}$ ), we write the production rate as

$$
Q(t)=\rho_{0} \frac{\varphi V_{R}}{\left\langle V_{S}\right\rangle}\left\langle\frac{V_{S}}{t_{c}} q_{K n}^{\prime}\left(t / t_{c}\right)\right\rangle=\frac{\rho_{0} \varphi V_{R}}{t_{c}^{*}}\left\langle\frac{\epsilon_{V_{s}}}{\epsilon_{t_{c}}} q_{K n}^{\prime}\left(t / t_{c}^{*} \epsilon_{t_{c} c}\right)\right\rangle,
$$

where $t_{c}^{*}$ is the characteristic time of the bundle (e.g. $t_{c}^{*}=\left\langle t_{c}\right\rangle$ or $t_{c}^{*}=\langle K n\rangle^{3} \times$ $\left.\left\langle V_{S}\right\rangle\langle L\rangle /\left(\pi \ell_{0}^{2} D_{0}^{m} / 4\right)\right)$ and we have defined the relative characteristic time and storage volume of a single tube as

$$
\epsilon_{t_{c}} \equiv \frac{t_{c}}{t_{c}^{*}} \quad \text { and } \quad \epsilon_{V_{s}} \equiv \frac{V_{S}}{\left\langle V_{S}\right\rangle}
$$

respectively. The recovery fraction is

$$
R(t)=\frac{1}{\rho_{0} \varphi V_{R}} \int_{0}^{t} Q(\tau) \mathrm{d} \tau=\left\langle\epsilon_{V_{s}} \int_{0}^{t / t_{c}} q_{K n}^{\prime}\left(\tau^{\prime}\right) \mathrm{d} \tau^{\prime}\right\rangle=\left\langle\epsilon_{V_{s}} R_{K n, t_{c}}\left(t / t_{c}^{*}\right)\right\rangle
$$

where

$$
R_{K n, \epsilon_{t_{c}}}\left(t^{\prime}\right)=\int_{0}^{t^{\prime} / \epsilon_{t_{c}}} q_{K n}^{\prime}\left(\tau^{\prime}\right) \mathrm{d} \tau^{\prime}=\int_{0}^{t^{\prime}} \frac{q_{K n}^{\prime}\left(\tau^{\prime} / \epsilon_{t_{c}}\right)}{\epsilon_{t_{c}}} \mathrm{~d} \tau^{\prime} .
$$

Notice that in BoDTM only the Knudsen number modifies the shape of the dimensionless production rate from a single tube, $q_{K n}^{\prime}\left(t^{\prime}\right)$, whereas the characteristic time and the storage volume only affect the relative scaling of the solutions for different tubes. When the production rate and the recovery fraction are calculated numerically, the average operator is approximated from $N_{t}$ tubes sampled from the distribution,

$$
\langle *\rangle \approx \frac{1}{N_{t}} \sum_{i=1}^{N_{t}} *
$$

In practice, to avoid computing the production rate for too many tubes, a discrete distribution can be assumed for the Knudsen number. 


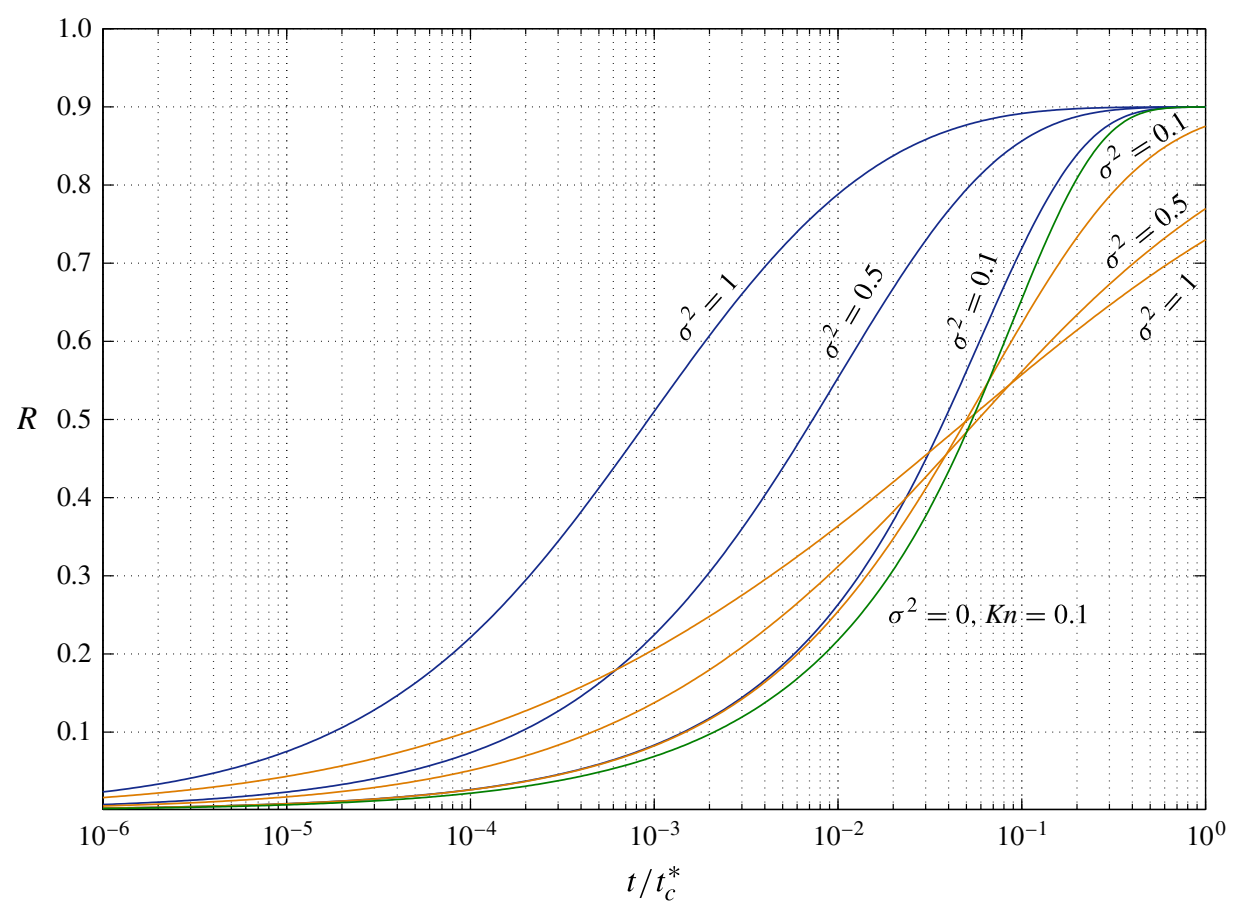

FIgURE 8. (Colour online) Recovery fraction, $R$, as a function of the dimensionless time, $t / t_{c}^{*}$, for heterogeneous bundles of dual tubes with different variance and correlation model. Blue curves correspond to a perfect correlation between $K n$ and $V_{S}$, i.e. $\epsilon_{V_{S}}=$ $V_{S} /\left\langle V_{S}\right\rangle=K n^{-2} /\langle K n\rangle^{-2}$, whereas orange curves correspond to no correlation between the two quantities. In both cases, $K n$ and $V_{S}$ follow a log-normal distribution with variance $\sigma_{\log K n}^{2}=\sigma^{2}$ and $\sigma_{\log V_{S}}^{2}=4 \sigma^{2}\left(\sigma^{2}=0.1,0.5,1\right.$ as indicated in the figure $)$. All curves are obtained from a bundle of $N_{t}=10000$ dual tubes with $\langle K n\rangle=0.1$. Also shown is the recovery fraction for a homogeneous bundle $\left(\sigma^{2}=0, K n=0.1\right)$.

\subsection{Effects of heterogeneity and correlation model}

In order to investigate the effects of heterogeneity and statistical correlations, we consider idealized joint distribution functions. For simplicity, the tube length is assumed constant. First, we consider bundles of tubes in which storage volume and Knudsen number are either perfectly correlated or uncorrelated. In the perfectly correlated case, the storage diameter is proportional to the conductive diameter $(\omega$ is constant) and we have

$$
\epsilon_{V_{s}}=\frac{V_{S}}{\left\langle V_{S}\right\rangle}=\frac{K n^{-2}}{\langle K n\rangle^{-2}} \quad \text { and } \quad \epsilon_{t_{c}}=\frac{t_{c}}{t_{c}^{*}}=\frac{K n}{\langle K n\rangle} .
$$

In the uncorrelated case, we have

$$
\epsilon_{t c}=\frac{t_{c}}{t_{c}^{*}}=\frac{K n^{3} V_{S}}{\langle K n\rangle^{3}\left\langle V_{S}\right\rangle} .
$$

In both cases, $K n$ and $V_{S}$ obey a log-normal distribution with variance $\sigma_{\log K n}^{2}$ and $\sigma_{\log V_{S}}^{2}=4 \sigma_{\log K n}^{2}$, respectively. 


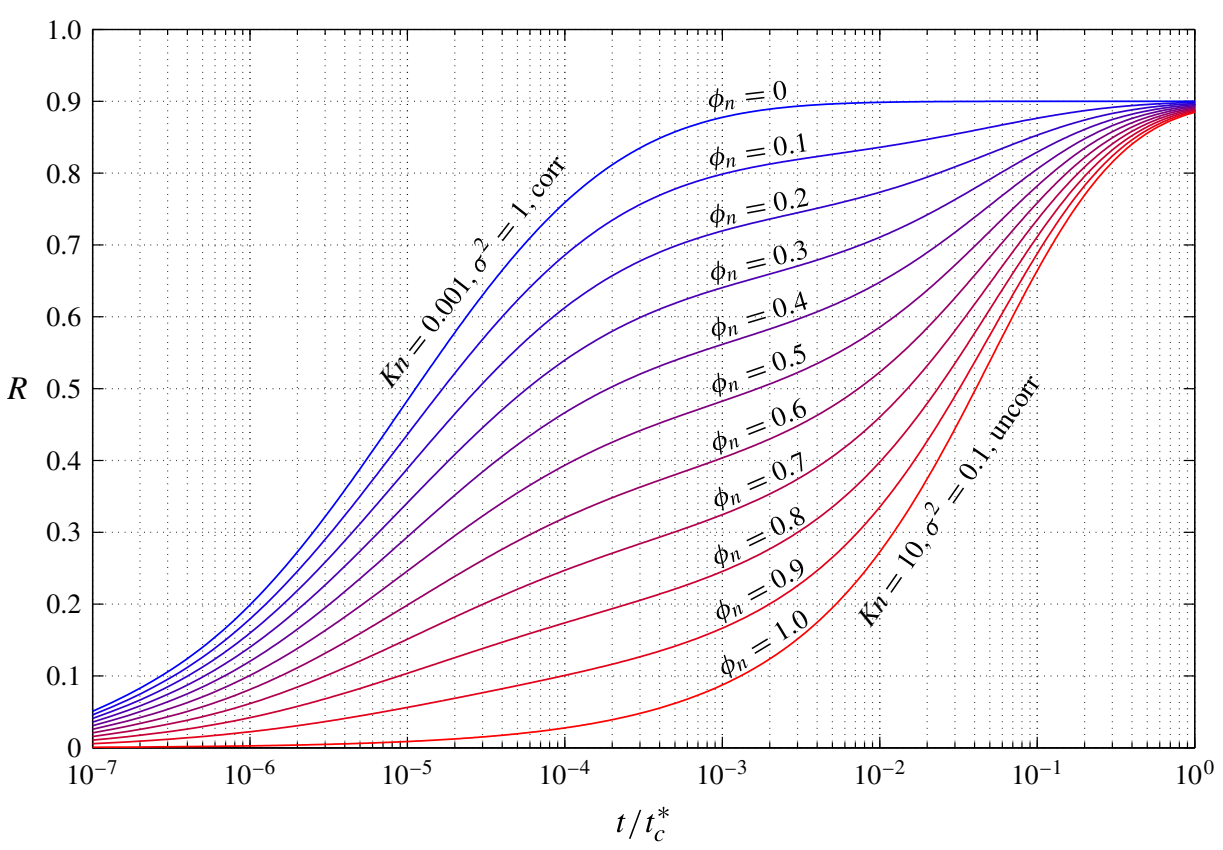

FIGURE 9. (Colour online) Recovery fraction, $R$, for a dual porosity shale matrix as a function of the dimensionless time, $t / t_{c}^{*}$. The red curve correspond to a nanoporosity characterized by $\langle K n\rangle=10$, variance $\sigma_{\log K n}^{2}=0.1$ and no correlation between $V_{S}$ and $K n$; the blue curve to a microporosity with $\langle K n\rangle=0.001$, variance $\sigma_{\log K n}^{2}=1$ and a perfect correlation between $V_{S}$ and $K n$. The intermediate curves correspond to dual porosity media in which the volume fraction of the nanoporosity, $\phi_{n}$, varies from 10 to $90 \%$. For each of the porosity types, a bundle of $N_{t}=10000$ tubes has been used.

The recovery fractions for different correlation models and variances are shown in figure 8 for $\langle K n\rangle=0.1$. In the case of perfect correlation, the recovery fraction systematically grows with the variance: more conductive pores contain larger volumes of gas and control the recovery, particularly at early time. At later time, smaller pores determine the production rate, but the volume of produced gas is small. A recovery fraction of 0.5 (respectively 0.8 ) is obtained 40 times (respectively 10 times) earlier for $\sigma^{2}=1$ than for $\sigma^{2}=0.1$. When storage volume and conductive diameter are uncorrelated, very heterogeneous bundles lead to higher recovery fraction at early time, but lower recovery at later time, owing to the effects of less conductive pores that can contain a considerable volume fraction. (Similar results, not shown here, have been obtained for different $\langle K n\rangle$.)

Multiporosity models can be quickly built from simple joint p.d.f.s, and the recovery fraction can be computed as

$$
R(t)=\sum_{i=1}^{m} \phi_{i} R_{i}(t),
$$

where $\phi_{i}$ and $R_{i}(t)$ are the porosity fraction and the recovery fraction of the $i$ porosity, respectively. As an example, we consider a dual-porosity shale matrix characterized by a mildly heterogeneous nanoporosity $\left(K n=10, \sigma_{\log K n}^{2}=0.1\right)$ with no correlation 
between conductive and storage volumes, and a strongly heterogeneous microporosity $\left(K n=0.001, \sigma_{\log K n}^{2}=1\right)$ with perfect correlation between $V_{S}$ and $K n$. The recovery for different fractions of the nanoporosity, $\phi_{n}$, varying between 0 and 1 , is shown in figure 9 and illustrates the flexibility of BoDTM, which can reproduce complex recovery behaviour by increasing the complexity of the joint p.d.f. that describes the porosity of the shale matrix.

\section{A simple comparison with field production data}

\subsection{Field production data from the Barnett shale-gas field}

Large-scale exploitation of shale gas started in the Barnett shale formation, which is located in North Texas, USA, and has been producing since 2003. As of today, the Barnett shale-gas field has the largest cumulative production and is one of the five fields that contribute to $80 \%$ of US shale gas (Hughes 2013a,b). Barnett production data have been analysed by several authors (e.g. Baihly et al. 2010; Patzek, Male \& Marder 2013; Pratikno, Reese \& Maguire 2013). Here, as an example of the application of BoDTM, we consider the average gas production rate from 25 wells that have been operated since 2003, as grouped in Baihly et al. (2010). This dataset has been chosen because late-time production deviates from the $t^{-\frac{1}{2}}$ decline of the production rate and this divergence is necessary to characterize the formation.

The average production rate obtained from Baihly et al. (2010) is plotted in figure 10 as a function of time. In general, early-time data suffer from the aftermath of drilling and hydrofracturing (e.g. cleaning up after completion, back-production of hydrofracturing water) or from constraints on maximum production rate and well loading (Patzek et al. 2013; Pratikno et al. 2013). Therefore, some time is required to establish the nonlinear diffusive regime described by our model and the production rate is typically below the $-\frac{1}{2}$ slope (on a log-log plot) at an early stage. This can be seen as a positive time offset, which sensitively affects only early-time data (see figure 10). At intermediate time, the average production rate is very well described by the $-\frac{1}{2}$ decline; whereas divergence from this behaviour is again observed at late time. From the experimental dataset, the divergence time is estimated as $t_{M}=34$ months when the production rate, expressed as normal cubic metres of gas per second, is $q_{M}=0.186 \mathrm{Nm}^{3} \mathrm{~s}^{-1}$ (6000 Mscf day ${ }^{-1}$ (Mscf $=$ thousand standard cubic feet per day)). (In this paper, we use SI units; when appropriate, the corresponding quantities in field units are given in parentheses to facilitate comparison with the reservoir engineering literature.)

\subsection{Production rate and cumulative production predicted by calibrated BoDTM}

As the production-rate record is too short to characterize the decline behaviour after the $-\frac{1}{2}$ decline, in the following we compare the field data with the results of different BoDTMs that are calibrated using $\left(t_{M}, q_{M}\right)$ as matching point. As in the previous section, we assume $\rho_{0} / \rho_{f}=0.1$ and we consider three homogeneous BoDTMs with $K n=0.001,0.1$ and 10. In addition, we also consider two heterogeneous BoDTMs with the same Knudsen number and variance $\left(K n=0.1\right.$ and $\sigma^{2}=0.1$, respectively) but different correlation between storage and conductive diameters (i.e. they are either perfectly correlated or uncorrelated, as described in §5.3). These five models are calibrated by matching their production rate at the time of divergence from the $-\frac{1}{2}$ decline to the field production rate $\left(q_{M}\right)$ at the time of divergence estimated from the data $\left(t_{M}\right)$. The production-rate curves of the calibrated models are plotted in 


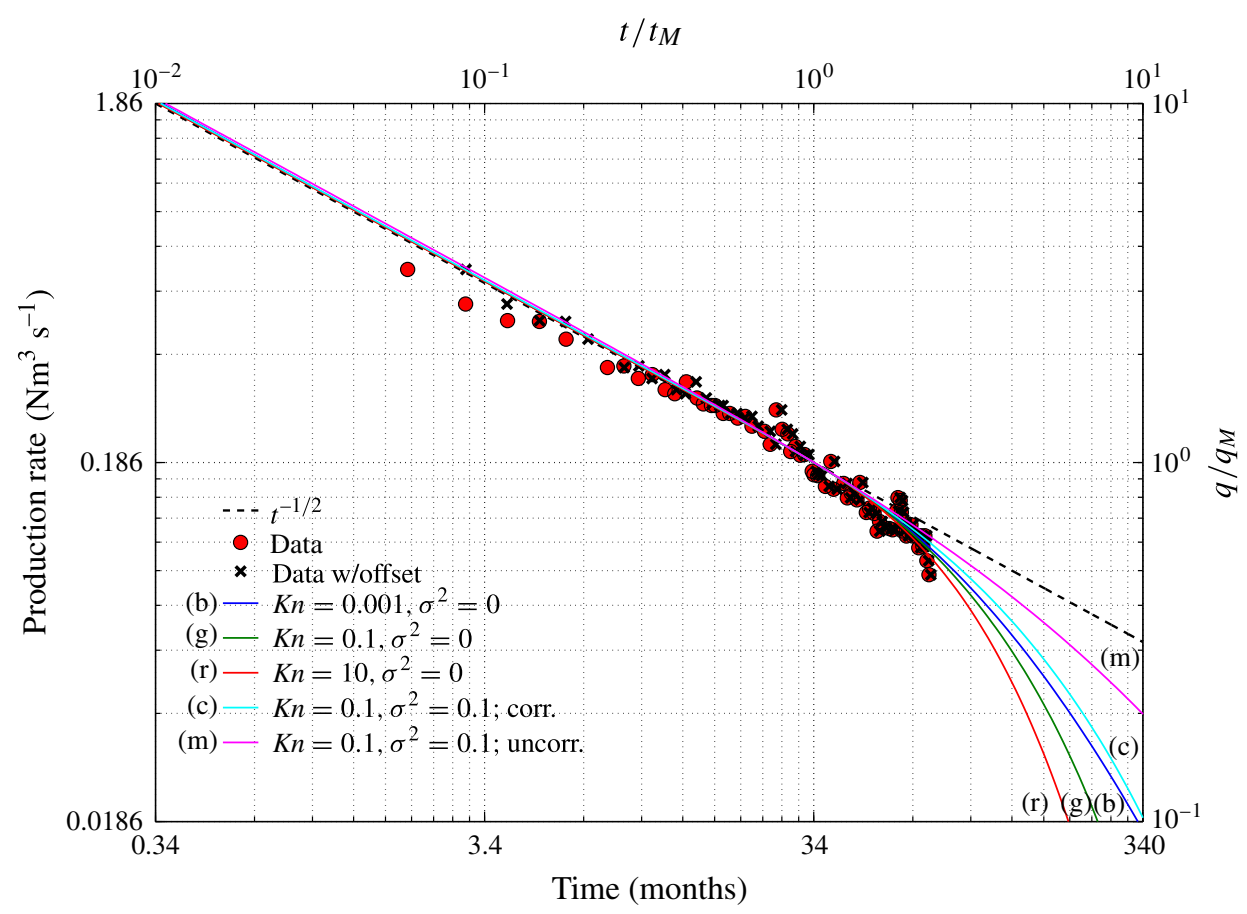

FIGURE 10. (Colour online) Average gas production rate from 25 wells in the Barnett shale formation as grouped in Baihly et al. (2010) (red circles). At intermediate time (10-30 months) the production decline is proportional to $t^{-\frac{1}{2}}$ (black dashed line), whereas at earlier time the production data are lower. A positive offset of one month can be used to correct the discrepancy without significantly affecting the late-time behaviour (black crosses). At later time, the divergence from the $-\frac{1}{2}$ decline can be estimated at $t_{M}=34$ months and the corresponding production rate is $q_{M}=0.186 \mathrm{Nm}^{3} \mathrm{~s}^{-1}$. The field data are compared with five BoDTMs that have been calibrated by matching the time of divergence from the $-\frac{1}{2}$ decline.

figure 10. Without any further assumption, from the values at the matching point, it is possible to estimate $M_{R}$, i.e. the amount of gas in the formation, which varies between $0.76 \times 10^{8} \mathrm{Nm}^{3}$ (2.83 Bscf) and $1.84 \times 10^{8} \mathrm{Nm}^{3}$ (6.87 Bscf) depending on the model employed (Bscf $=$ billion $\left(10^{9}\right)$ standard cubic feet).

The models exhibiting a quicker decline seem to better describe the field data in figure 10, but the time series is too short to clearly discriminate among models. Although all models match the time of divergence from the $-\frac{1}{2}$ decline, the subsequent behaviour is very different and leads to very different forecasts of the cumulative production (figure 11). The latter is sensitively affected by the Knudsen number and by the correlation model used to describe the statistics of the pore space; as a result, the predicted life span of the well can vary considerably depending on the BoDTM employed. This demonstrates that field production at later time can be correctly predicted only if we model all relevant transport mechanisms and describe the effects of possible statistical fluctuations of the shale properties.

Recently, Patzek et al. (2013) have also analysed the production of the Barnett shale-gas field with a nonlinear diffusion model. They only consider Darcy's flow because they assume that gas transport is dominated by the effective properties of 


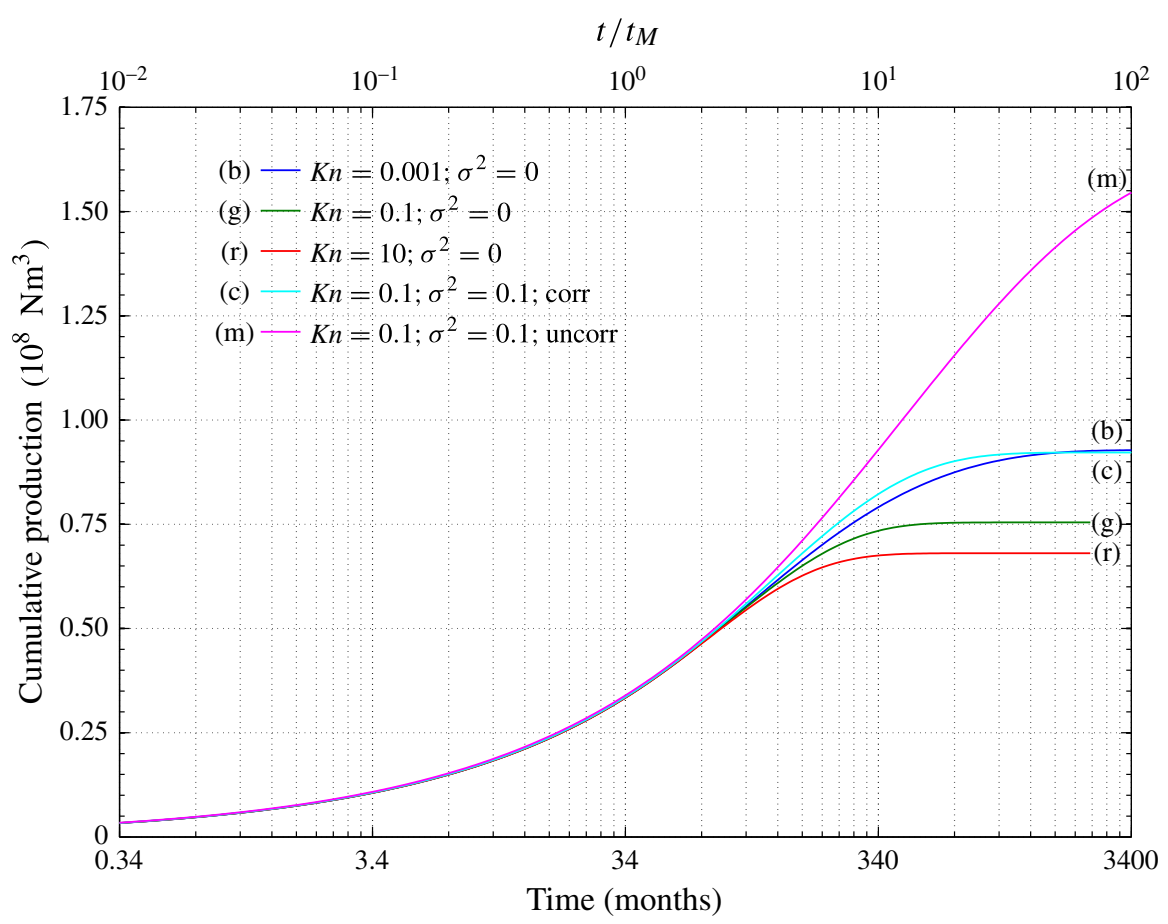

FIgURE 11. (Colour online) Cumulative production for five BoDTMs that have been calibrated to the field production data from the Barnett shale. Shown is the evolution as a function of time, both in dimensional units (bottom axis) and normalized by $t_{M}=$ 34 months, the time of divergence from the $-\frac{1}{2}$ decline (top axis). (See also figure 10.)

a fracture network. As the solution depends only weakly on the pressure difference between the formation and the hydraulic fractures, they essentially obtain a single decline curve (Patzek et al. 2013). However, as we have discussed in $\S 2.5$, most of the gas volume is stored in the undisturbed shale porosity and, as a first approximation, the production rate is constrained by the travel time from the shale matrix into the fracture network. A correct description of the transport mechanisms in the shale matrix requires the use of an effective diffusion coefficient that depends on the Knudsen number (4.8). Taking into account multiple transport mechanisms, we obtain a family of production curves that yield very different cumulative productions. The solutions of Patzek et al. (2013) are recovered for small $K n$ : our results for $K n=0.001$ are very similar to the production predicted by their curve for the pressure range 400-4000 p.s.i.a. (p.s.i.a. $=$ pounds per square inch, absolute), which corresponds to a density ratio $\rho_{f} / \rho_{0}=0.106$.

\subsection{Physical and geometric parameters of the formation}

In the following, we calculate the geometric parameters of the fractured shale formation with realistic physical parameters. We suppose that the shale formation is initially at pressure $p_{0}=27.6 \mathrm{MPa}(4000$ p.s.i.a. $)$ and temperature $T_{0}=93.3^{\circ} \mathrm{C}$ $\left(200^{\circ} \mathrm{F}\right)$, which are of the order of the parameters usually encountered in the reservoir engineering literature (e.g. Patzek et al. 2013; Pratikno et al. 2013). At these conditions, the natural gas (methane) has density $\rho_{0}=140 \mathrm{~kg} \mathrm{~m}^{-3}$, mean free path 
(a) Physical parameters
$T_{0}\left({ }^{\circ} \mathrm{C}\right)$
$p_{0}(\mathrm{MPa})$
$\rho_{0}\left(\mathrm{~kg} \mathrm{~m}^{-3}\right)$
$D_{0}^{m}\left(\mathrm{~m}^{2} \mathrm{~s}^{-1}\right)$
$\ell_{0}(\mathrm{~nm})$
$\varphi(\%)$
93.3
27.6
140
$6.92 \times 10^{-8}$
0.30
6.0

(b) Geometric parameters

$\begin{array}{ccccccc}\text { Model } & \sigma^{2} & K n & M_{R}\left(\mathrm{Nm}^{-3}(\mathrm{Bscf})\right) & V_{R}\left(\mathrm{~m}^{3}\right) & d(\mathrm{~m}) & L / \sqrt{\omega}(\mathrm{m}) \\ \text { Homogeneous } & 0.0 & 0.001 & 1.04 \times 10^{8}(3.86) & 0.89 \times 10^{7} & 3.0 \times 10^{-7} & 3360 \\ \text { Homogeneous } & 0.0 & 0.1 & 0.84 \times 10^{8}(3.14) & 0.72 \times 10^{7} & 3.0 \times 10^{-9} & 42.9 \\ \text { Homogeneous } & 0.0 & 10 & 0.76 \times 10^{8}(2.83) & 0.65 \times 10^{7} & 3.0 \times 10^{-11} & 4.29 \\ \text { Corr. } & 0.1 & 0.1 & 1.03 \times 10^{8}(3.82) & 0.88 \times 10^{7} & 3.0 \times 10^{-9} & 63.2 \\ \text { Uncorr. } & 0.1 & 0.1 & 1.84 \times 10^{8}(6.87) & 1.58 \times 10^{7} & 3.0 \times 10^{-9} & 110\end{array}$

TABLE 2. Physical parameters of the shale formation and geometric parameters from the five calibrated BoDTMs. The formation is initially at temperature $T_{0}$ and pressure $p_{0}$. Here $\rho_{0}, D_{0}^{m}$ and $\ell_{0}$ are the corresponding density, molecular diffusion and mean free path of the natural gas; and $\varphi$ is the porosity of the formation. For each BoDTM (which is described by the heterogeneity model, by the variance, $\sigma^{2}$, and by the Knudsen number, $K n$ ), we report the gas mass in the formation estimated from the calibration, $M_{R}$, as well as the calculated formation volume, $V_{R}$, the conductive diameter, $d$, and the effective length, $L^{e}=$ $L / \sqrt{\omega}$.

$\ell_{0}=0.30 \mathrm{~nm}$ from (3.5) and molecular diffusion coefficient $D_{0}^{m}=6.92 \times 10^{-8} \mathrm{~m}^{2} \mathrm{~s}^{-1}$ from (3.7). Using these physical parameters, we can calculate a set of geometric parameters of the formation, which are reported in table 2 for each BoDTM. In particular, for the models that seem to better fit the data, the formation volume from which the gas is produced, $V_{R}=\rho_{N} M_{R} / \rho_{0} \varphi$, is in excellent agreement with the typical hydrofractured shale volume per well, which is around $10^{7} \mathrm{~m}^{3}$ (Pratikno et al. 2013). The conductive diameter can be readily computed from the Knudsen number, $d=\ell_{0} / K n$, and the block length, $L_{B}$, can be estimated from $L^{e}=L / \sqrt{\omega}=\theta L_{B} / \sqrt{\omega}$, which is a sort of effective length that takes into account the effects of the tortuosity, $\theta$, and of the ratio between conductive and storage radius, $\sqrt{\omega}=d / d_{S}$ (table 2). For the high reservoir pressure and temperature considered here, the model assuming predominant viscous flow $(K n=0.001)$ leads to unrealistically high permeability $(k=2.81 \mathrm{mD})$ and effective block length; whereas the typical pore size for the pure Knudsen regime $(K n=10)$ is unrealistically small. The three models with a Knudsen number in the transition regime $(K n=0.1)$ lead to more realistic diameters and to effective lengths, $L^{e}$, that are consistent with a block size, $L_{B}$, of the order of a metre if the effects of $\theta$ and $\omega$ are taken into account.

\section{Discussion and conclusions}

To model gas transport in shale formations, we have introduced an effective diffusion that describes advection with slippage effects, Knudsen diffusion and self-diffusion. In dimensionless form, the nonlinear coefficient contains only one parameter, $\mathrm{Pe}_{*}$, which is a reduced Péclet number depending on the equation of state, on the molecular kinetic model of the fluid and on the geometric factor that characterizes the pore space. Using elementary kinetics, this parameter depends only on the dimensionless permeability, $\kappa$, and can be approximate by $\frac{1}{9}$ for a tube and by $\frac{2}{7}$ for a fracture. Therefore, for a structural feature with defined geometry, 
the relationship between the nonlinear dimensionless diffusion coefficient and the dimensionless density is described by a single curve. It is important that this coefficient incorporates both Knudsen diffusion and self-diffusion because at reservoir conditions the density is high and intermolecular collisions dominate wall collisions. Also, at intermediate density the combined effects of wall and molecular collisions reduce the diffusion coefficient, which exhibits a minimum.

The dimensionless diffusion coefficient can be used in standard Darcy-scale reservoir models, eventually transformed into a dimensional nonlinear apparent permeability of the porous matrix. In this approach, the network of microseismic fractures and other structural features that characterize the shale formation after hydraulic fracturing have to be modelled explicitly. Here, following a different strategy, we have proposed an approximate model to estimate the production rate. Based on the observation that the undisturbed porous matrix has much smaller effective diffusion coefficient and much larger storage capacity than the fractures, the residence time in the fracture system can be neglected. This greatly simplifies the problem of estimating the production rate, which can be approximated as the flow from the shale matrix into the secondary fracture network and calculated with a BoDTM.

The bundle comprises dual tubes that are characterized by a conductive and a storage diameter; they provide a conceptualization of gas pathways that consist of sequences of bulbs and throats. The conductive diameter is controlled by small throats (bottlenecks), whereas the storage diameter is determined by the larger pores, in which the largest amount of gas is stored. The dual tubes allow us to model systems characterized by slow recovery of large gas volumes. By simply introducing a dependence of the diameters on gas density (or pressure), the model can incorporate geomechanical effects on the porosity and Langmuir-isotherm adsorption, which can be important in very small pores at comparatively low pressures.

We have shown that the single-tube solution, which is the basis of the BoDTM, is approximately self-similar at early time, but not at later time when the gas flux declines exponentially due to the finite length of the tube. The production rate can be estimated with a bundle sampled from the joint distribution of the dual tubes, which has to be inferred from the available information on the pore geometry. As it naturally accounts for statistical variability, the BoDTM is very flexible and can easily include different porosity and correlation models. We have shown, for simplified distributions, that the effects of pathway variability strongly depend on the degree of correlation between conductive and storage diameters. Complex production rates can be modelled by combining bundle statistics from simple joint distributions. This suggests that more complex pore network models (e.g. the bulb-and-throat model) are not necessary to estimate complex production rates.

The effectiveness and flexibility of our approach is also supported by comparison with field production data from the Barnett shale-gas field. Although the $-\frac{1}{2}$ decline observed in field production data can be modelled by any nonlinear diffusion equation, we have demonstrated that the specific form of the effective diffusion coefficient determines the late-time behaviour. Some models appear to fit equally well the early production data, but predict very different cumulative production at later time. This demonstrates the importance of characterizing the relevant physical processes (transport mechanisms and multiporosity effects, as discussed in this paper, but also adsorption and geomechanics that have not been considered for simplicity). It is important to complement field production data with independent information from field or laboratory measurements in order to constrain the parameters of the model 
(e.g. the Knudsen number and the statistics of the pore-size distribution). Assuming realistic physical parameters for the shale formation, we have shown that the calibrated BoDTM can estimate the fractured shale volume per well. The estimated pore size suggests that the production takes place in a transition regime and that self-diffusion is an important transport mechanism.

The model presented here is based on several approximations, but our framework is very flexible and allows for extensions of increasing complexity, which can be successively employed when required or when more data become available. As mentioned above, geomechanics effects or Langmuir adsorption can be included by employing density-dependent tube diameters. Also, more refined gas dynamic models can be employed to compute the reduced Péclet number or the compressibility factor. If necessary, the travel time in the fracture system can be taken into account by employing, together with the bundle of tubes representing the porosity of the shale matrix, a bundle of fractures. The same nonlinear diffusion coefficient can be used but with a different value of the reduced Péclet number, $P e_{*}$. The bundle of fractures and the bundle of tubes can be in series, or can continuously interact as in a dual porosity model with exchange term. The fact the BoDTM is based on a limited number of parameters is also an advantage when the model is used in history matching or optimization problems.

\section{Acknowledgements}

The authors are grateful to Dr W. Narr for providing helpful suggestions; to Dr R. Salazar-Tio and Dr B. Sun for helping to obtain X-ray scanning pictures of micro/nanopores; and to $\mathrm{Dr} \mathrm{P}$. Tomin for interesting discussions. This work was supported in part by Chevron Energy Technology Company. I.L. is Swiss National Science Foundation Professor at the University of Lausanne (grant numbers SNSF PP00P2-144922/1 and PP00P2-123419/1).

\section{Appendix A. Derivation of the dimensionless diffusion equation}

From the general mass-balance equation (3.1), the transversely integrated gasbalance equation can be written in the form

$$
\frac{\pi d_{S}^{2}}{4} \frac{\partial \rho}{\partial t}=\frac{\pi d^{2}}{4} \frac{\partial}{\partial x}\left\{\rho \frac{k}{\mu} \frac{\partial p}{\partial x}+D \frac{\partial \rho}{\partial x}\right\}=\frac{\pi d^{2}}{4} \frac{\partial}{\partial x}\left\{\left[\rho \frac{k}{\mu} \frac{\partial p}{\partial \rho}+D\right] \frac{\partial \rho}{\partial x}\right\},
$$

where the dependence of the pressure on the density, $p=p(\rho)$, is given by the equation of state. Assuming that the process is approximately isothermal (3.20), we have $(\partial p / \partial \rho)_{T_{0}} \approx\left(p_{0} / \rho_{0}\right) \zeta(\rho)$. Using the definition of the permeability that accounts for slippage effects (3.17),

$$
k=\left[1+4\left(K n \rho_{0} / \rho\right)\right] \kappa d^{2},
$$

and the definition of the total diffusion coefficient that includes self-diffusion and Knudsen diffusion ((4.12) with $\gamma=1)$,

$$
D=D^{m}\left[1+\left(K n \rho_{0} / \rho\right)\right]^{-1},
$$

we obtain

$$
\frac{d_{S}^{2}}{d^{2}} \frac{\partial \rho}{\partial t}=\frac{\partial}{\partial x}\left\{\left[\frac{\rho}{\rho_{0}} \frac{\kappa d^{2} p_{0}}{\mu} \zeta(\rho)\left(1+4 K n \rho_{0} / \rho\right)+\frac{D^{m}}{1+K n \rho_{0} / \rho}\right] \frac{\partial \rho}{\partial x}\right\} .
$$


For an isothermal process, we have $\mu=\mu_{0}$ and $D^{m}=\rho_{0} / \rho D_{0}^{m}$ from (3.14) and (3.8), respectively, and we can write

$$
\frac{d_{S}^{2}}{d^{2}} \frac{\partial \rho}{\partial t}=\frac{\partial}{\partial x}\left\{\left[\frac{\rho}{\rho_{0}} \frac{\kappa d^{2} p_{0}}{D_{0}^{m} \mu_{0}} \zeta(\rho)\left(1+4 K n \rho_{0} / \rho\right)+\frac{\rho_{0} / \rho}{1+K n \rho_{0} / \rho}\right] D_{0}^{m} \frac{\partial \rho}{\partial x}\right\}
$$

Then, using the definitions in (4.5),

$$
\omega=\frac{d^{2}}{d_{S}^{2}} \quad \text { and } \quad P e=\frac{\kappa d^{2} p_{0}}{D_{0}^{m} \mu_{0}}
$$

we obtain (4.1):

$$
\frac{1}{\omega} \frac{\partial \rho}{\partial t}=\frac{\partial}{\partial x}\left\{\left[\operatorname{Pe}(\rho)\left(\frac{\rho}{\rho_{0}}+4 K n\right)+\frac{1}{\frac{\rho}{\rho_{0}}+K n}\right] D_{0}^{m} \frac{\partial \rho}{\partial x}\right\} .
$$

For simplicity, we will assume that the compressibility factor varies sufficiently slowly with the density such that $\zeta(\rho) \approx 1$ (3.20). Using the relationship $P e=P e_{*} K n^{-2}$ (which is derived from kinetic theory, see appendix B) and after some algebraic manipulations, we obtain the nonlinear diffusion equation

$$
\frac{1}{\omega} \frac{\partial \rho}{\partial t}=\frac{\partial}{\partial x}\left\{D_{d i m}^{e}\left(\rho / \rho_{0} K n, P e_{*}, D_{0}^{m} / K n\right) \frac{\partial \rho}{\partial x}\right\},
$$

where the diffusion coefficient is

$$
D_{\text {dim }}^{e}\left(\rho / \rho_{0} K n, P e_{*}, D_{0}^{m} / K n\right)=\left[P e_{*}\left(\frac{\rho}{\rho_{0} K n}+4\right)+\frac{1}{\frac{\rho}{\rho_{0} K n}+1}\right] \frac{D_{0}^{m}}{K n} .
$$

With the definition of the dimensionless variables in (4.7),

$$
\rho^{\prime}=\frac{\rho}{\rho_{0}}, \quad x^{\prime}=\frac{x}{L} \quad \text { and } \quad t^{\prime}=\frac{t}{t_{c}}=\frac{\omega}{K n} \frac{D_{0}^{m}}{L^{2}} t,
$$

we have

$$
\frac{1}{\omega} \frac{\omega D_{0}^{m}}{K n L^{2}} \frac{\partial \rho^{\prime}}{\partial t^{\prime}}=\frac{D_{0}^{m}}{K n L^{2}} \frac{\partial}{\partial x^{\prime}}\left\{\left[P e_{*}\left(\rho^{\prime} / K n+4\right)+\frac{1}{\rho^{\prime} / K n+1}\right] \frac{\partial \rho^{\prime}}{\partial x^{\prime}}\right\},
$$

which simplifies to (4.8) with the definition of the effective diffusion coefficient in (4.12),

$$
D^{e}\left(\rho^{\prime} / K n, P e_{*}\right)=P e_{*}\left(\rho^{\prime} / K n+4\right)+\frac{1}{\rho^{\prime} / K n+1} .
$$

The (dimensional) diffusion coefficient can be written in terms of the (dimensionless) effective diffusion as

$$
D_{d i m}^{e}\left(\rho / \rho_{0} K n, P e_{*}, D_{0}^{m} / K n\right)=\frac{D_{0}^{m}}{K n} D^{e}\left(\rho / \rho_{0} K n, P e_{*}\right) .
$$


If the gas-balance equation is written in terms of pressure gradient rather than density gradient,

$$
\frac{1}{p_{0} \omega} \frac{\partial p}{\partial t}=\frac{\partial}{\partial x}\left\{\frac{k_{d i m}^{e}\left(p / p_{0} K n, P e_{*}, k\right)}{\mu_{0}} \frac{\partial p}{\partial x}\right\}
$$

the effective permeability is

$$
k_{d i m}^{e}\left(p / p_{0} K n, P e_{*}, k / K n\right)=\frac{k}{K n} D^{e}\left(p / p_{0} K n, P e_{*}\right)=\frac{\left(D_{0}^{m}\right)^{2}}{K n} \frac{\rho_{0}}{p_{0}} D^{e}\left(p / p_{0} K n, P e_{*}\right),
$$

where $k=\kappa d^{2}$ is the Darcy permeability.

\section{Appendix B. Reduced Péclet number}

From the definition of the Schmidt number, (3.12), and the Péclet number, (4.5), we can write

$$
P e=\frac{\kappa d^{2} p_{0}}{D_{0}^{m} \mu_{0}}=\frac{\kappa \ell_{0}^{2} p_{0}}{D_{0}^{m} \mu_{0}} \frac{d^{2}}{\ell_{0}^{2}}=P e_{*} K n^{-2},
$$

where the reduced Péclet number is

$$
P e_{*}=\frac{\kappa \ell_{0}^{2} p_{0}}{D_{0}^{m} \mu_{0}}=\frac{4 \kappa p_{0} \ell_{0}^{2}}{\xi_{D} \xi_{\mu} v_{T_{0}}^{2}},
$$

where we have used (3.7) and (3.13). From the equation of state (3.18) and the definition of the thermal velocity (3.6), we obtain

$$
p_{0}=Z_{0} \rho_{0} \frac{k_{B} T_{0}}{m}=Z_{0} \rho_{0} \frac{\pi}{8} v_{T_{0}}^{2},
$$

which can be inserted into (B 2) to obtain

$$
P e_{*}=\frac{\pi}{2} \frac{Z_{0} \kappa}{\xi_{\mu} \xi_{D}}
$$

Assuming $Z_{0} \approx 1$, for $\xi_{D}=\xi_{\mu}=\frac{2}{3}$, we have $P e_{*}=\frac{9 \pi}{256} \approx \frac{1}{9}$ for a tube and $P e_{*}=\frac{3 \pi}{32} \approx \frac{2}{7}$ for a fracture; whereas $\xi_{D}=\frac{5}{16} \pi$ and $\xi_{\mu}=\frac{3}{8} \pi$ yield $P e_{*}=\frac{2}{15 \pi} \approx \frac{1}{24}$ and $P e_{*}=\frac{16}{45 \pi} \approx \frac{6}{53}$ for a tube and a fracture, respectively.

\section{REFERENCES}

Baihly, J., Altman, R., Malpani, R. \& Luo, F. 2010 Shale gas production decline trend comparison over time and basins. In Proceedings of the SPE Annual Technical Conference and Exhibition, Florence, Italy, SPE 135555.

Bear, J. 1972 Dynamics of Fluids in Porous Media. Elsevier.

BJøRLYKKe, K. 2010 Petroleum Geoscience: From Sedimentary Environments to Rock Physics. Springer.

Blunt, M. J., Bijeljic, B., Dong, H., Gharbi, O., Iglauer, S., Mostaghimi, P., Paluszny, A. \& Pentland, C. 2013 Pore-scale imaging and modeling. Adv. Water Resour. 51, 197-216. 
Blunt, M. \& King, P. 1990 Macroscopic parameters for simulation of pore scale flow. Phys. Rev. A 42, 4780-4787.

Brown, G. P., Dinardo, A., Cheng, G. K. \& Sherwood, T. K. 1946 The flow of gases in pipes at low pressures. J. Appl. Phys. 17, 802-813.

Chapman, S. \& Cowling, T. G. 1939 The Mathematical Theory of Nonuniform Gases. Cambridge University Press.

Cui, X., Bustin, A. M. M.\& Bustin, R. M. 2009 Measurements of gas permeability and diffusivity of tight reservoir rocks: different approaches and their application. Geofluids 9, 208-223.

CURTiss, C. F. \& HirschFelder, J. O. 1949 Transport properties of multicomponent gas mixtures. J. Chem. Phys. 17, 550-555.

Darabi, H., Ettehad, A., Javadpour, F. \& Sepehrnoori, K. 2012 Gas flow in ultra-tight shale strata. J. Fluid Mech. 710, 641-658.

Dong, H. 2007 Micro/CT imaging and pore network extraction. PhD thesis, Imperial College, London.

EPSTEIN, N. 1989 On tortuosity and the tortuosity factor in flow and diffusion through porous media. Chem. Engng Sci. 44, 777-779.

FATT, I. 1956 The network model of porous media: 3. Dynamic properties of networks with tube size distribution. Trans. AIME 207, 164-169.

Gale, J. 2011 FRAC Consortium Meeting, Bureau of Economic Geology, University of Texas, Austin, $T X$.

Hirschfelder, J., Curtiss, C. F. \& Bird, R. B. 1954 Molecular Theory of Gases and Liquids. Wiley.

Hughes, J. D. $2013 a$ Drill, baby, drill: can unconventional fuels usher in a new era of energy abundance? Tech Rep. Post Carbon Institute.

Hughes, J. D. $2013 b$ A reality check on the shale revolution. Nature 494, 307-308.

JAVADPOUR, F. 2009 Nanopores and apparent permeability of gas flow in mudrocks (shales and siltstone). J. Can. Petrol. Technol. 48, 16-21.

Koplik, J. 1982 Creeping flow in two-dimensional networks. J. Fluid Mech. 119, 219-247.

Leahy-Dios, A., Das, M., Agarwal, A. \& Kaminsky, R. D. 2011 Modeling of transport phenomena and multicomponent sorption for shale gas and coalbed methane in an unsaturated grid simulator. In Proceedings of the SPE Annual Technical Conference and Exhibition, Denver, CO, SPE 147352.

Lee, S. H., Lough, M. F. \& Jensen, C. L. 2001 Hierarchical modeling of flow in naturally fractured formations with multiple length scales. Water Resour. Res. 37, 443-455.

Lee, S. H., Padmanabhan, L. \& Al-Sunaidi, H. 1996 Simulation of linear displacement experiments on massively parallel computers. SPE J. 1, 327-340.

Lough, M. F., Lee, S. H. \& Kamath, J. 1998 An efficient boundary integral formulation for flow through fractured porous media. J. Comput. Phys. 143, 462-483.

MaXWell, J. C. 1879 On stresses in rarified gases arising from inequalities of temperature. Phil. Trans. R. Soc. Lond. 170, 231-256.

MaXwell, S. \& Norton, M. 2012 Enhancing shale gas reservoir characterization using hydraulic fracture microseismic data. First Break 30, 95-101.

Meyers, A. L. \& Monson, P. A. 2002 Adsorption in porous materials at high pressure: theory and experiment. Langmuir 18, 10261-10273.

Patzek, T. W., Male, F. \& Marder, M. 2013 Gas production in the Barnett shale obeys a simple scaling theory. Proc. Natl Acad. Sci. USA 110, 19731-19736.

Poling, B. E., Prausnitz, J. M. \& O'Connell, J. P. 2000 Properties of Gases and Liquids. McGraw-Hill.

Pratikno, H., Reese, D. E. \& Maguire, M. M. 2013 Production analysis in the Barnett shale - field example for reservoir characterization using public data. In Proceedings of the SPE Annual Technical Conference and Exhibition, New Orleans, LS, SPE 166176.

Roy, S., Raju, R., Chuang, H. F., Cruden, B. A. \& Meyyappan, M. 2003 Modeling gas flow through microchannels and nanopores. J. Appl. Phys. 93, 4870-4879. 
SArkar, S., Jupe, A., LAngan, R. \& Rosca, A. 2012 Uncertainty in microseismic event source locations associated with a fracture stimulation in a tight sand. In Proceedings of the Istanbul International Geophysical Conferences and Oil \& Gas Exhibition, Istanbul, Turkey.

Swami, V. \& SetTari, A. 2012 A pore scale gas flow model for shale gas reservoir. In Proceedings of the Americas Unconventional Resources Conference, Pittsburgh, PA, SPE 155756.

US Energy Information Administration 2013a Annual Energy Outlook 2013. Washington, DC. http://www.eia.gov/forecasts/aeo/pdf/0383(2013).pdf.

US Energy Information Administration 2013b Technically Recoverable Shale Oil and Shale Gas Resources. Washington, DC. http://www.eia.gov/analysis/studies/worldshalegas/.

VÁzquez, J. L. 2007 The Porous Medium Equation - Mathematical Theory. Clarendon.

VERMYLEN, J. P. \& ZOBACK, M. 2011 Hydraulic fracturing, microseismic magnitudes and stress evolution in the Barnett shale, Texas, USA. In Proceedings of the SPE Hydraulic Fracturing Technology Conference and Exhibition, The Woodlands, TX, SPE 140507.

WALls, J. D. \& Sinclair, S. W. 2011 Eagle Ford shale reservoir properties from digital rock physics. First Break 29, 97-101.

Welty, J. R., Wicks, C. E., Wilson, R. E. \& Rorrer, G. L. 2007 Fundamentals of Momentum, Heat, and Mass Transfer. Wiley.

Zoback, M., Kitasei, S. \& Copithorne, B. July 2010 Addressing the Environmental Risks from Shale Gas Development. Worldwatch Institute. 